TRANSACTIONS OF THE

AMERICAN MATHEMATICAL SOCIETY

Volume 354, Number 10, Pages 4131-4151

S 0002-9947(02)03039-8

Article electronically published on June 4, 2002

\title{
WEAK AMENABILITY OF MODULE EXTENSIONS OF BANACH ALGEBRAS
}

\author{
YONG ZHANG
}

\begin{abstract}
We start by discussing general necessary and sufficient conditions for a module extension Banach algebra to be $n$-weakly amenable, for $n=0,1,2, \cdots$. Then we investigate various special cases. All these case studies finally provide us with a way to construct an example of a weakly amenable Banach algebra which is not 3-weakly amenable. This answers an open question raised by H. G. Dales, F. Ghahramani and N. Grønbæk.
\end{abstract}

\section{INTRODUCTION}

Suppose that $\mathfrak{A}$ is a Banach algebra, and that $X$ is a Banach $\mathfrak{A}$-bimodule. A derivation from $\mathfrak{A}$ into $X$ is a linear operator $D: \mathfrak{A} \rightarrow X$ satisfying

$$
D(a b)=D(a) b+a D(b) \quad(a, b \in \mathfrak{A}) .
$$

A derivation $D$ is inner if there is $x_{0} \in X$ such that $D(a)=a x_{0}-x_{0} a$ for $a \in \mathfrak{A}$. The quotient space $\mathcal{H}^{1}(\mathfrak{A}, X)$ of all continuous derivations from $\mathfrak{A}$ into $X$ modulo the subspace of inner derivations is called the first cohomology group of $\mathfrak{A}$ with coefficients in $X$. A Banach algebra $\mathfrak{A}$ is said to be amenable if $\mathcal{H}^{1}\left(\mathfrak{A}, X^{*}\right)=\{0\}$ for every Banach $\mathfrak{A}$-bimodule $X$; here $X^{*}$ denotes the Banach dual module of $X$. The algebra $\mathfrak{A}$ is said to be weakly amenable if $\mathcal{H}^{1}\left(\mathfrak{A}, \mathfrak{A}^{*}\right)=\{0\}$, and is called $n$-weakly amenable, for an integer $n \geq 0$, if $\mathcal{H}^{1}\left(\mathfrak{A}, \mathfrak{A}^{(n)}\right)=\{0\}$, where $\mathfrak{A}^{(n)}$ is the $n$-th dual module of $\mathfrak{A}$ when $n \geq 1$, and is $\mathfrak{A}$ itself when $n=0$. The algebra $\mathfrak{A}$ is said to be permanently weakly amenable if it is $n$-weakly amenable for all $n \geq 1$.

The concept of weak amenability was first introduced by Bade, Curtis and Dales in 1 for commutative Banach algebras, and was extended to the noncommutative case by Johnson in [22] (see also [7, 9], 11]-[16], 21] and [24]). Dales, Ghahramani and Grønbæk initiated the study of $n$-weak amenability of Banach algebras in their recent paper [10], where they revealed many important properties of this sort of Banach algebra. An interesting problem concerning this class of Banach algebras is the relation between $n$-weak amenability and $m$-weak amenability for different integers $n$ and $m$. For instance, if $\mathfrak{A}$ is a commutative Banach algebra, then the assertion that $\mathfrak{A}$ is weakly amenable is equivalent to saying that it is permanently weakly amenable ([1 Theorem 1.5]); but, for noncommutative Banach algebras, things are different - we only know that $(n+2)$-weak amenability implies $n$-weak

Received by the editors August 23, 1999 and, in revised form, January 25, 2002.

2000 Mathematics Subject Classification. Primary 46H20; Secondary 47B47, 46H10, 46H25, $46 \mathrm{H} 35$.

Key words and phrases. $n$-weakly amenable, module, dual module, derivation, operator algebra.

Research supported by NSERC. 
amenability for $n \geq 1$ ([10, Proposition 1.2]), and weak amenability does not imply 2 -weak amenability ([10, Theorems 5.1 and 5.2]). After investigating varieties of classical Banach algebras, Dales, Ghahramani and Grønbæk raised and left open the following question in [10]: Does weak amenability imply 3 -weak amenability?

This paper is designed to answer the preceding question. We will construct a counterexample to the question. For this purpose, we study $n$-weak amenability of the module extension Banach algebra $\mathfrak{A} \oplus X$, the $l_{1}$-direct sum of a Banach algebra $\mathfrak{A}$ and a nonzero Banach $\mathfrak{A}$-module $X$ with the algebra product defined as follows:

$$
(a, x) \cdot(b, y)=(a b, a y+x b) \quad(a, b \in \mathfrak{A}, x, y \in X) .
$$

Some aspects of algebras of this form have been discussed in [2] and [10. We choose this class of Banach algebras to investigate for the preceding question because this class is neither too small nor is it too large; it contains permanently weakly amenable Banach algebras (see Section 6), and it contains no amenable Banach algebras due to [8, Lemma 2.7], since $X$ is a complemented nilpotent ideal in the algebra. If $\mathfrak{A}$ has both left and right approximate identities and they are also, respectively, left and right approximate identities for $X$, then $\mathfrak{A} \oplus X$ cannot be pointwise approximately biprojective (see [30]). The class of module extension Banach algebras also includes the natural triangular Banach algebra whose amenability has been investigated in 12. We will give some comment on the latter algebra in Section 2 .

This paper is organized as follows: in Section 1 we study the construction of module actions of $2 m$-th dual algebras on $2 m$-th dual modules. This extends the corresponding discussion in [10]. In Section 2 we give the main theorems which deal with the necessary and sufficient conditions for $\mathfrak{A} \oplus X$ to be $n$-weakly amenable. Section 3 discusses various techniques for lifting derivations. These will be applied in Section 4 to give the proofs of the main theorems. Sections 5 and 6 deal with the special cases of $X=\mathfrak{A}, \mathfrak{A}^{*}$ and $X_{0}$, where $X_{0}$ denotes an $\mathfrak{A}$-bimodule with the right module action trivial. In Section 7 we first discuss the condition for $\mathfrak{A} \oplus\left(X_{1} \dot{+} X_{2}\right)$ to be weakly amenable, where $\dot{+}$ denotes the $l_{1}$ direct sum (of modules). Then, we give an example of a weakly amenable Banach algebra of this form and prove that it is not 3-weakly amenable. This finally answers the preceding open question in the negative.

Since $(\mathfrak{A} \oplus X)^{*}=(0 \oplus X)^{\perp} \dot{+}(\mathfrak{A} \oplus 0)^{\perp}$, where $\dot{+}$ denotes the direct $\mathfrak{A}$-module $l_{\infty}$-sum, and $(0 \oplus X)^{\perp}$ (respectively, $\left.(\mathfrak{A} \oplus 0)^{\perp}\right)$ is isometrically isomorphic to $\mathfrak{A}^{*}$ (respectively, $X^{*}$ ) as $\mathfrak{A}$-bimodules, for convenience, in this paper we simply identify the corresponding terms and write:

$$
(\mathfrak{A} \oplus X)^{*}=\mathfrak{A}^{*}+X^{*} .
$$

Similarly, we will identify the underlying space of the $n$-th conjugate $(\mathfrak{A} \oplus X)^{(n)}$ with $\mathfrak{A}^{(n)} \dot{+} X^{(n)}$. The sum is an $l_{1}$-sum when $n$ is even and is an $l_{\infty}$-sum when $n$ is odd.

\section{Bimodule aCtions of $\mathfrak{A}^{(2 m)}$ ON $X^{(2 m)}$}

Suppose that $\mathfrak{A}$ is a Banach algebra, and $X$ is a Banach $\mathfrak{A}$-bimodule. According to [10, pp. 27 and 28], $X^{* *}$ is a Banach $\mathfrak{A}^{* *}$-bimodule, where $\mathfrak{A}^{* *}$ is equipped with the first Arens product. The module actions are successively defined as follows. 
First, for $x \in X, f \in X^{*}, \phi \in X^{* *}$ and $u \in \mathfrak{A}^{* *}$, define $\phi f, f x \in \mathfrak{A}^{*}$ and $u f \in X^{*}$ by

$$
\begin{gathered}
\langle a, \phi f\rangle=\langle f a, \phi\rangle, \quad\langle a, f x\rangle=\langle x a, f\rangle \quad(a \in \mathfrak{A}), \\
\langle x, u f\rangle=\langle f x, u\rangle \quad(x \in X) .
\end{gathered}
$$

Then, for $\phi \in X^{* *}$ and $u \in \mathfrak{A}^{* *}$, define $u \phi, \phi u \in X^{* *}$ by

$$
\langle f, u \phi\rangle=\langle\phi f, u\rangle, \quad\langle f, \phi u\rangle=\langle u f, \phi\rangle \quad\left(f \in X^{*}\right) .
$$

These give the left and right $\mathfrak{A}^{* *}$-module actions on $X^{* *}$. Also, the definition for $u f$ with $u \in \mathfrak{A}^{* *}$ and $f \in X^{*}$ gives a left Banach $\mathfrak{A}^{* *}$-module action on $X^{*}$. When $u=a \in \mathfrak{A}$, all the above $\mathfrak{A}^{* *}$-module actions agree with the $\mathfrak{A}$-module actions on the corresponding dual modules $X^{*}$ and $X^{* *}$. Moreover, it is readily seen that, with these module actions, the first Arens product on $(\mathfrak{A} \oplus X)^{* *}$ may be represented by

$$
(u, \phi) \cdot(v, \psi)=(u v, u \psi+\phi v) \quad\left(u, v \in \mathfrak{A}^{* *}, \phi, \psi \in X^{* *}\right) .
$$

Viewing $\mathfrak{A}^{(2 m)}$ as a new $\mathfrak{A}$ and $X^{(2 m)}$ as a new $X$, the preceding procedure will successively define $X^{(2 m+2)}$ as a Banach $\mathfrak{A}^{(2 m+2)}$-bimodule. Here, and throughout the paper, the first Arens product is consistently assumed on each $\mathfrak{A}^{(2 n)}$. Since some relations arising from the procedure are important for later use, we now give the definition in detail as follows.

Suppose that the bimodule action of $\mathfrak{A}^{(2 m)}$ on $X^{(2 m)}$ has been defined, where $m \geq 1$. Then in a natural way, $X^{(2 m+k)}, k \geq 1$, is a Banach $\mathfrak{A}^{(2 m)}$-bimodule with the module multiplications $u \Lambda$ and $\Lambda u \in X^{(2 m+k)}$, for $\Lambda \in X^{(2 m+k)}$ and $u \in \mathfrak{A}^{(2 m)}$, defined by

$$
\langle\gamma, u \Lambda\rangle=\langle\gamma u, \Lambda\rangle, \quad\langle\gamma, \Lambda u\rangle=\langle u \gamma, \Lambda\rangle \quad\left(\gamma \in X^{(2 m+k-1)}\right)
$$

If $u=a \in \mathfrak{A}$, these module actions coincide with $\mathfrak{A}$-module actions on $X^{(2 m+k)}$.

Then, for $F \in X^{(2 m+1)}$ and $\Phi \in X^{(2 m+2)}$, define $F \Phi, \Phi F \in \mathfrak{A}^{(2 m+1)}$ by

$$
\langle u, F \Phi\rangle=\langle F, \Phi u\rangle(=\langle u F, \Phi\rangle)
$$

and

$$
\langle u, \Phi F\rangle=\langle F u, \Phi\rangle(=\langle F, u \Phi\rangle) \quad\left(u \in \mathfrak{A}^{(2 m)}\right) .
$$

Throughout this paper, for a Banach space $Y$ and an element $y \in Y, \hat{y}$ always denotes the image of $y$ in $Y^{* *}$ under the canonical mapping. When $F \in X^{(2 m+1)}$ and $\phi \in X^{(2 m)}$, we denote $F \hat{\phi}$ by $F \phi$ and $\hat{\phi} F$ by $\phi F$. It is easy to check that

$$
\langle u, F \phi\rangle=\langle\phi u, F\rangle, \quad\langle u, \phi F\rangle=\langle u \phi, F\rangle \quad \text { for } u \in \mathfrak{A}^{(2 m)} .
$$

By using the canonical image of $F$ or $\Phi$ in the appropriate $2 l$-th dual space of the space that it belongs to, we can then signify a meaning for $F \Phi$ and $\Phi F$ for every $F \in$ $X^{(2 m+1)}$ and $\Phi \in X^{(2 n)}$; they are elements of $\mathfrak{A}^{(2 k+1)}$, where $k=\max \{m, n-1\}$.

Now for $\mu \in \mathfrak{A}^{(2 m+2)}$ and $F \in X^{(2 m+1)}$, we define $\mu F \in X^{(2 m+1)}$ by

$$
\langle\phi, \mu F\rangle=\langle F \phi, \mu\rangle \quad\left(\phi \in X^{(2 m)}\right) .
$$

This actually defines a left Banach $\mathfrak{A}^{(2 m+2)}$-module action on $X^{(2 m+1)}$.

Finally, for $\mu \in \mathfrak{A}^{(2 m+2)}$ and $\Phi \in X^{(2 m+2)}$, define $\mu \Phi, \Phi \mu \in X^{(2 m+2)}$ by

$$
\langle F, \mu \Phi\rangle=\langle\Phi F, \mu\rangle, \quad\langle F, \Phi \mu\rangle=\langle\mu F, \Phi\rangle \quad\left(F \in X^{(2 m+1)}\right) .
$$

These finally define the $\mathfrak{A}^{(2 m+2)}$-module actions on $X^{(2 m+2)}$ and, therefore, complete our definition. 
If $\lim u_{\alpha}=\mu$ in $\left.\sigma\left(\mathfrak{A}^{(2 m+2)}\right), \mathfrak{A}^{(2 m+1)}\right)$ and $\lim \phi_{\beta}=\Phi$ in $\sigma\left(X^{(2 m+2)}, X^{(2 m+1)}\right)$, where $\left(u_{\alpha}\right) \subset \mathfrak{A}^{(2 m)}$ and $\left(\phi_{\beta}\right) \subset X^{(2 m)}$, and $\sigma\left(Y^{*}, Y\right)$ denotes the weak ${ }^{*}$ topology on $Y^{*}$, then

$$
\mu \Phi=\lim _{\alpha} \lim _{\beta} u_{\alpha} \phi_{\beta}, \quad \Phi \mu=\lim _{\beta} \lim _{\alpha} \phi_{\beta} u_{\alpha} \quad \text { in } \sigma\left(X^{(2 m+2)}, X^{(2 m+1)}\right) .
$$

For $\mu \in \mathfrak{A}^{(2 m+2)}$ and $\phi \in X^{(2 m)}$, since $\mu \phi=\mu \hat{\phi}, \phi \mu=\hat{\phi} \mu$, we have

$$
\langle F, \mu \phi\rangle=\langle\phi F, \mu\rangle,\langle F, \phi \mu\rangle=\langle F \phi, \mu\rangle \quad\left(F \in X^{(2 m+1)}\right) .
$$

One can also easily check the relations

$$
\begin{gathered}
u \hat{f}=\hat{u} \hat{f}=(u f)^{\wedge}, \\
\hat{f} \hat{\phi}=(f \phi)^{\wedge}, \quad \hat{\phi} \hat{f}=(\phi f)^{\wedge}, \\
\hat{u} \hat{\phi}=(u \phi)^{\wedge}, \quad \hat{\phi} \hat{u}=(\phi u)^{\wedge},
\end{gathered}
$$

where $f \in X^{(2 m-1)}, \phi \in X^{(2 m)}$ and $u \in \mathfrak{A}^{(2 m)}(m \geq 1)$. Therefore, each product agrees with those previously defined.

Concerning dual module morphisms, we have the following.

Lemma 1.1. Suppose that $X$ and $Y$ are Banach $\mathfrak{A}$-bimodules. Then, for every continuous $\mathfrak{A}$-bimodule morphism $\tau: X \rightarrow Y$ and for each $m \geq 1, \tau^{(2 m)}: X^{(2 m)} \rightarrow$ $Y^{(2 m)}$, the $2 m$-th dual operator of $\tau$ is an $\mathfrak{A}^{(2 m)}$-bimodule morphism.

Proof. It suffices to prove the lemma in the case where $m=1$. However, for this simple case, the proof is straightforward if we note that $\tau^{* *}$ is weak ${ }^{*}$-weak ${ }^{*}$ continuous.

In the following, to avoid involving unnecessarily complicated notation, for an element $y$ in a Banach space $Y$, we will use the same notation $y$ to represent its canonical image in any of the $2 m$-th dual spaces $Y^{(2 m)}$.

Take $\mathfrak{A}^{(n)} \dot{+} X^{(n)}$ as the underlying space of $(\mathfrak{A} \oplus X)^{(n)}$. From induction, by using the relations in (1.1) and (1.2), one can verify that the $(\mathfrak{A} \oplus X)$-bimodule actions on $(\mathfrak{A} \oplus X)^{(n)}$ are formulated as follows:

$$
(a, x) \cdot\left(a^{(n)}, x^{(n)}\right)= \begin{cases}\left(a a^{(n)}+x x^{(n)}, a x^{(n)}\right), & \text { if } n \text { is odd } \\ \left(a a^{(n)}, a x^{(n)}+x a^{(n)}\right), & \text { if } n \text { is even }\end{cases}
$$

and

$$
\left(a^{(n)}, x^{(n)}\right) \cdot(a, x)= \begin{cases}\left(a^{(n)} a+x^{(n)} x, x^{(n)} a\right), & \text { if } n \text { is odd; } \\ \left(a^{(n)} a, a^{(n)} x+x^{(n)} a\right), & \text { if } n \text { is even, }\end{cases}
$$

where $(a, x) \in \mathfrak{A} \oplus X$ and $\left(a^{(n)}, x^{(n)}\right) \in \mathfrak{A}^{(n)} \dot{+} X^{(n)}=(\mathfrak{A} \oplus X)^{(n)}$.

\section{MAIN THEOREMS}

Suppose that $\mathfrak{A}$ is a Banach algebra, and $X$ is a Banach $\mathfrak{A}$-bimodule. For $n$-weak amenability of the Banach algebra $\mathfrak{A} \oplus X$, we have the following main results, whose proofs will be given in Section 4.

Theorem 2.1. For $m \geq 0, \mathfrak{A} \oplus X$ is $(2 m+1)$-weakly amenable if and only if the following conditions hold:

1. $\mathfrak{A}$ is $(2 m+1)$-weakly amenable;

2. $\mathcal{H}^{1}\left(\mathfrak{A}, X^{(2 m+1)}\right)=\{0\}$; 
3. for every continuous $\mathfrak{A}$-bimodule morphism $\Gamma: X \rightarrow \mathfrak{A}^{(2 m+1)}$, there is $F \in$ $X^{(2 m+1)}$ such that $a F-F a=0$ for $a \in \mathfrak{A}$ and $\Gamma(x)=x F-F x$ for $x \in X$;

4. the only continuous $\mathfrak{A}$-bimodule morphism $T: X \rightarrow X^{(2 m+1)}$ for which $x T(y)$ $+T(x) y=0(x, y \in X)$ in $\mathfrak{A}^{(2 m+1)}$ is $T=0$.

Theorem 2.2. For $m \geq 0, \mathfrak{A} \oplus X$ is $2 m$-weakly amenable if and only if the following conditions hold:

1. the only continuous derivations $D: \mathfrak{A} \rightarrow \mathfrak{A}^{(2 m)}$ for which there is a continuous operator $T: X \rightarrow X^{(2 m)}$ such that $T(a x)=D(a) x+a T(x)$ and $T(x a)=$ $x D(a)+T(x) a(a \in \mathfrak{A}, x \in X)$ are the inner derivations;

2. $\mathcal{H}^{1}\left(\mathfrak{A}, X^{(2 m)}\right)=\{0\}$;

3. the only continuous $\mathfrak{A}$-bimodule morphism $\Gamma: X \rightarrow \mathfrak{A}^{(2 m)}$ for which $x \Gamma(y)$ $+\Gamma(x) y=0(x, y \in X)$ in $X^{(2 m)}$ is zero;

4. for every continuous $\mathfrak{A}$-bimodule morphism $T: X \rightarrow X^{(2 m)}$, there exists $u \in \mathfrak{A}^{(2 m)}$ for which au = ua for $a \in \mathfrak{A}$ and $T(x)=x u-u x$ for $x \in X$.

Remark 2.3. A simple calculation shows that, when $m=0$, condition 3 in Theorem 2.1 is equivalent to the following:

$3^{0}$. there is no nonzero continuous $\mathfrak{A}$-bimodule morphism $\Gamma: X \rightarrow \mathfrak{A}^{*}$.

For the general case, condition 3 in Theorem 2.1 is equivalent to the following:

$3^{m}$. if $\Gamma: X \rightarrow \mathfrak{A}^{(2 m+1)}$ is a continuous $\mathfrak{A}$-bimodule morphism, then $\Gamma(X) \subset \mathfrak{A}^{\perp}$ and there is $G \in X^{(2 m+1)} \cap X^{\perp}$ for which $a G-G a=0$ in $X^{(2 m+1)}(a \in \mathfrak{A})$ and $\Gamma(x)=x G-G x(x \in X)$.

Proposition 2.4. Suppose that condition 4 of Theorem 2.1 holds for an $m \geq 0$. Then, $\operatorname{span}(\mathfrak{A} X+X \mathfrak{A})$ is dense in $X$.

Proof. Assume, towards a contradiction, that $\operatorname{span}(\mathfrak{A} X+X \mathfrak{A})$ is not dense in $X$. Take a nonzero element $F \in X^{*} \cap(\mathfrak{A} X+X \mathfrak{A})^{\perp}$, and define $T: X \rightarrow X^{*}$ by

$$
T(x)=F(x) F .
$$

Since $\left.F\right|_{\mathfrak{A} X+X \mathfrak{A}}=0$, it is easy to see that $T$ is a nonzero, continuous $\mathfrak{A}$-bimodule morphism and that $\mathfrak{A} T(X)=T(X) \mathfrak{A}=\{0\}$. Also, for $x, y \in X$, we have $x T(y)=$ $T(x) y=0$ in $\mathfrak{A}^{*}$ since $T(X) \subset(\mathfrak{A} X)^{\perp} \cap(X \mathfrak{A})^{\perp}$. This shows that condition 4 of Theorem 2.1 does not hold for $m=0$. So it does not hold for all $m \geq 0$. This is a contradiction.

Corollary 2.5. For $m=0$, condition 4 in Theorem 2.1 is equivalent to the following:

$4^{0} \cdot \operatorname{span}(\mathfrak{A} X+X \mathfrak{A})$ is dense in $X$ and there is no nonzero $\mathfrak{A}$-bimodule morphism $T: X \rightarrow X^{*}$ satisfying $\langle x, T(y)\rangle+\langle y, T(x)\rangle=0$ for $x, y \in X$.

Proof. Suppose that condition 4 in Theorem 2.1 holds. From the preceding proposition, $\operatorname{span}(\mathfrak{A} X+X \mathfrak{A})$ is dense in $X$. If the $\mathfrak{A}$-bimodule morphism $T: X \rightarrow X^{*}$ satisfies

$$
\langle x, T(y)\rangle+\langle y, T(x)\rangle=0 \quad \text { for } x, y \in X,
$$

then, for every $a \in \mathfrak{A}$,

$$
\langle a, x T(y)+T(x) y\rangle=\langle a x, T(y)\rangle+\langle y, T(a x)\rangle=0 .
$$

This shows that $x T(y)+T(x) y=0$ for $x, y \in X$. Therefore, $T=0$ and so $4^{0}$ holds. 
Conversely, if $4^{0}$ holds, and $T: X \rightarrow X^{*}$ is a continuous $\mathfrak{A}$-bimodule morphism satisfying $x T(y)+T(x) y=0$ in $\mathfrak{A}^{*}$, then, for every $x=a x_{1}+x_{2} b \in \mathfrak{A} X+X \mathfrak{A}$ and $y \in X$, we have

$$
\langle x, T(y)\rangle+\langle y, T(x)\rangle=\left\langle a, x_{1} T(y)+T\left(x_{1}\right) y\right\rangle+\left\langle b, T(y) x_{2}+y T\left(x_{2}\right)\right\rangle=0 .
$$

Since $\operatorname{span}(\mathfrak{A} X+X \mathfrak{A})$ is dense in $X$, this implies that $\langle x, T(y)\rangle+\langle y, T(x)\rangle=0$ for all $x, y \in X$. Hence $T=0$, and so condition 4 of Theorem 2.1 holds for $m=0$.

Suppose that $\mathfrak{A}$ and $\mathfrak{B}$ are Banach algebras, and let $\mathcal{M}$ be a Banach $\mathfrak{A}, \mathfrak{B}$-module. The algebra $\mathcal{T}$ with the triangular matrix structure

$$
\mathcal{T}=\left(\begin{array}{cc}
\mathfrak{A} & \mathcal{M} \\
0 & \mathfrak{B}
\end{array}\right)
$$

is called a triangular Banach algebra. The sum and product on $\mathcal{T}$ are given by the usual $2 \times 2$ matrix operations and obvious internal module actions. The norm on $\mathcal{T}$ is

$$
\left\|\left(\begin{array}{cc}
a & m \\
0 & b
\end{array}\right)\right\|=\|a\|_{\mathfrak{A}}+\|m\|_{\mathcal{M}}+\|b\|_{\mathfrak{B}} .
$$

Denote by $\mathfrak{A}+\mathfrak{B}$ the direct $l_{1}$-sum Banach algebra of $\mathfrak{A}$ and $\mathfrak{B}$, and view $\mathcal{M}$ as an $(\mathfrak{A}+\mathfrak{B})$-bimodule with the module actions given by

$$
(a, b) \cdot m=a m, \quad m \cdot(a, b)=m b, \quad a \in \mathfrak{A}, b \in \mathfrak{B}, m \in M .
$$

Then $\mathcal{T}$ is isometrically isomorphic to the module extension Banach algebra $(\mathfrak{A}+\mathfrak{B})$ $\oplus \mathcal{M}$. With this setting and some calculations, one sees that Theorems 2.1 and 2.2 imply some main results in [12]. For instance, if $\mathfrak{A}$ and $\mathfrak{B}$ are unital and $\mathcal{M}$ is a unital $\mathfrak{A}, \mathfrak{B}$-module, then $\mathcal{T}$ is weakly amenable if and only if both $\mathfrak{A}$ and $\mathfrak{B}$ are weakly amenable. In fact, the condition can be weakened further to the following: there exist a bounded approximate identity of $\mathfrak{A}$ and a bounded approximate identity of $\mathfrak{B}$ that are also, respectively, left and right approximate identities for $\mathcal{M}$.

\section{LiFTING DERIVATIONS}

In this section we give several lemmas concerning the lifting of derivations (and module morphisms) from $\mathfrak{A}$ (or $X)$ into $\mathfrak{A}^{(n)}$ or $X^{(n)}$ to derivations from $\mathfrak{A} \oplus X$ into $(\mathfrak{A} \oplus X)^{(n)}$.

Lemma 3.1. Suppose that $\Gamma: X \rightarrow \mathfrak{A}^{(2 m+1)}$ is a continuous $\mathfrak{A}$-bimodule morphism. Then $\bar{\Gamma}: \mathfrak{A} \oplus X \rightarrow(\mathfrak{A} \oplus X)^{(2 m+1)}$, defined by

$$
\bar{\Gamma}((a, x))=(\Gamma(x), 0),
$$

is a continuous derivation. The derivation $\bar{\Gamma}$ is inner if and only if there exists $F \in X^{(2 m+1)}$ such that $a F-F a=0$ and $\Gamma(x)=x F-F x$ for $a \in \mathfrak{A}$ and $x \in X$.

Proof. It is straightforward to check that $\bar{\Gamma}$ is a continuous derivation. Noting that $(\Gamma(x), 0)=\bar{\Gamma}((0, x))$ and $\bar{\Gamma}((a, 0))=(0,0)$, one can also see easily that the element $F \in \mathfrak{A}^{(2 m+1)}$ described in the lemma exists if $\bar{\Gamma}$ is inner.

Conversely, if such an element $F$ exists, then

$$
\bar{\Gamma}((a, x))=(\Gamma(x), 0)=(x F-F x, a F-F a)=(a, x) \cdot(0, F)-(0, F) \cdot(a, x),
$$

showing that $\bar{\Gamma}$ is inner. 
A similar proof gives the following lemma.

Lemma 3.2. Suppose that $T: X \rightarrow X^{(2 m)}$ is a (continuous) $\mathfrak{A}$-bimodule morphism. Then $\bar{T}: \mathfrak{A} \oplus X \rightarrow(\mathfrak{A} \oplus X)^{(2 m)}$, defined by

$$
\bar{T}((a, x))=(0, T(x)),
$$

is a continuous derivation. The derivation $\bar{T}$ is inner if and only if there exists $u \in \mathfrak{A}^{(2 m)}$ such that $u a=a u$ for $a \in \mathfrak{A}$, and $T(x)=x u-u x$ for all $x \in X$.

Concerning dual operators we have the following.

Lemma 3.3. Suppose that $k>0$ is an integer, and that $D: \mathfrak{A} \rightarrow X^{(k)}$ is a (continuous) derivation. Then, for every integer $m \geq 0, D^{(2 m+1)}: X^{(k+2 m+1)} \rightarrow \mathfrak{A}^{(2 m+1)}$, the $(2 m+1)$-th dual operator of $D$, satisfies

$$
\begin{aligned}
& D^{(2 m+1)}(a F)=a D^{(2 m+1)}(F)-\left.(D(a) F)\right|_{\mathfrak{A}^{(2 m)},}, \\
& D^{(2 m+1)}(F a)=D^{(2 m+1)}(F) a-\left.(F D(a))\right|_{\mathfrak{A}^{(2 m)},}
\end{aligned}
$$

for $a \in \mathfrak{A}$ and $F \in X^{(k+2 m+1)}$.

Proof. The lemma is true for $m=0$ because

$$
\left\langle b, D^{*}(a F)\right\rangle=\langle D(b) a, F\rangle=\langle D(b a)-b D(a), F\rangle=\left\langle b, a D^{*}(F)-D(a) F\right\rangle
$$

and

$$
\left\langle b, D^{*}(F a)\right\rangle=\langle a D(b), F\rangle=\langle D(a b)-D(a) b, F\rangle=\left\langle b, D^{*}(F) a-F D(a)\right\rangle,
$$

for $a, b \in \mathfrak{A}$ and $F \in X^{(k+1)}$.

For $m>0$, from Proposition 1.7 of [10], $D^{(2 m)}: \mathfrak{A}^{(2 m)} \rightarrow X^{(k+2 m)}$ is a (continuous) derivation; here we take the first Arens product in each $\mathfrak{A}^{(2 m)}$. Then, the above shows that $D^{(2 m+1)}=\left(D^{(2 m)}\right)^{*}: X^{(k+2 m+1)} \rightarrow\left(\mathfrak{A}^{(2 m)}\right)^{*}$ satisfies

$$
D^{(2 m+1)}(u F)=u D^{(2 m+1)}(F)-\left.\left(D^{(2 m)}(u) F\right)\right|_{\mathfrak{A}(2 m)}
$$

and

$$
D^{(2 m+1)}(F u)=D^{(2 m+1)}(F) u-\left.\left(F D^{(2 m)}(u)\right)\right|_{\mathfrak{A}(2 m)},
$$

for $u \in \mathfrak{A}^{(2 m)}$ and $F \in X^{(k+2 m+1)}$. In particular, when $u=a \in \mathfrak{A}$, these give the formulae in the lemma.

Lemma 3.4. Let $m$ be an integer. Suppose that $D: \mathfrak{A} \rightarrow X^{(2 m+1)}$ is a (continuous) derivation. Then $\bar{D}: \mathfrak{A} \oplus X \rightarrow(\mathfrak{A} \oplus X)^{(2 m+1)}$, defined by

$$
\bar{D}((a, x))=\left(-D^{(2 m+1)}(x), D(a)\right) \quad \text { for }(a, x) \in \mathfrak{A} \oplus X,
$$

is also a (continuous) derivation. Moreover,

1. if $\bar{D}$ is inner, then so is $D$;

2. if $D$ is inner, then there exists a (continuous) derivation $\widetilde{D}: \mathfrak{A} \oplus X \rightarrow$ $(\mathfrak{A} \oplus X)^{(2 m+1)}$ satisfying $\widetilde{D}((a, 0))=0(a \in \mathfrak{A})$ and for which $\bar{D}-\widetilde{D}$ is inner. 
Proof. For $a, b \in \mathfrak{A}$ and $x, y \in X$, we have, from Lemma 3.3

$$
\begin{aligned}
\bar{D}((a, x) \cdot(b, y))=\bar{D}((a b, a y+x b))=\left(-D^{(2 m+1)}(a y+x b), D(a b)\right) \\
=\left(-\left[a D^{(2 m+1)}(y)-\left.(D(a) y)\right|_{\mathfrak{A}^{(2 m)}}\right.\right. \\
\left.\left.\quad \quad+D^{(2 m+1)}(x) b-\left.(x D(b))\right|_{\mathfrak{A}^{(2 m)}}\right], D(a) b+a D(b)\right) \\
=\left(-\left[a D^{(2 m+1)}(y)-D(a) y+D^{(2 m+1)}(x) b-x D(b)\right], D(a) b+a D(b)\right) \\
=\left(-a D^{(2 m+1)}(y)+x D(b), a D(b)\right)+\left(-D^{(2 m+1)}(x) b+D(a) y, D(a) b\right) \\
=(a, x) \cdot\left(-D^{(2 m+1)}(y), D(b)\right)+\left(-D^{(2 m+1)}(x), D(a)\right) \cdot(b, y) \\
=(a, x) \cdot \bar{D}((b, y))+\bar{D}((a, x)) \cdot(b, y) .
\end{aligned}
$$

Therefore, $\bar{D}$ is a (continuous) derivation.

If $\bar{D}$ is inner, then, for some $u \in \mathfrak{A}^{(2 m+1)}$ and $F \in X^{(2 m+1)}$, we have

$$
\bar{D}((a, x))=(a, x) \cdot(u, F)-(u, F) \cdot(a, x) .
$$

Thus,

$$
(0, D(a))=\bar{D}((a, 0))=(a, 0) \cdot(u, F)-(u, F) \cdot(a, 0)=(a u-u a, a F-F a) .
$$

This shows that $D(a)=a F-F a$ for all $a \in \mathfrak{A}$, and hence $D$ is inner.

Conversely, if $D$ is inner, then there exists $F \in X^{(2 m+1)}$ such that $D(a)=$ $a F-F a$ for $a \in \mathfrak{A}$. Let $T: X \rightarrow \mathfrak{A}^{(2 m+1)}$ be defined by

$$
T(x)=-D^{(2 m+1)}(x)-(x F-F x) \quad(x \in X),
$$

and let $\bar{T}: \mathfrak{A} \oplus X \rightarrow(\mathfrak{A} \oplus X)^{(2 m+1)}$ be defined by

$$
\bar{T}((a, x))=(T(x), 0) \quad((a, x) \in \mathfrak{A} \oplus X) .
$$

Then

$$
(\bar{D}-\bar{T})((a, x))=(x F-F x, a F-F a)=(a, x) \cdot(0, F)-(0, F) \cdot(a, x)
$$

for $(a, x) \in \mathfrak{A} \oplus X$. Therefore, $\bar{D}-\bar{T}$ is an inner derivation. This in turn implies that $\bar{T}$ is a (continuous) derivation. So $\widetilde{D}=\bar{T}$ satisfies all the requirements. This completes the proof.

If $D$ is a (continuous) derivation from $\mathfrak{A}$ into $\mathfrak{A}^{(2 m+1)}, m \geq 0$, we define $\bar{D}$ : $\mathfrak{A} \oplus X \rightarrow(\mathfrak{A} \oplus X)^{(2 m+1)}$ by

$$
\bar{D}((a, x))=(D(a), 0) .
$$

If $D$ is a (continuous) derivation from $\mathfrak{A}$ into $X^{(2 m)}, m \geq 0$, we define $\bar{D}: \mathfrak{A} \oplus X \rightarrow$ $(\mathfrak{A} \oplus X)^{(2 m)}$ by

$$
\bar{D}((a, x))=(0, D(a)) .
$$

If $T: X \rightarrow \mathfrak{A}^{(2 m)}$ is a (continuous) $\mathfrak{A}$-bimodule morphism, satisfying $x T(y)+$ $T(x) y=0$ in $X^{(2 m)}$ for $x, y \in X$, we define $\bar{T}: \mathfrak{A} \oplus X \rightarrow(\mathfrak{A} \oplus X)^{(2 m)}$ by

$$
\bar{T}((a, x))=(T(x), 0) .
$$

Finally, if $T: X \rightarrow X^{(2 m+1)}$ is a (continuous) $\mathfrak{A}$-bimodule morphism, satisfying $x T(y)+T(x) y=0$ for $x, y \in X$, we define $\bar{T}: \mathfrak{A} \oplus X \rightarrow(\mathfrak{A} \oplus X)^{(2 m+1)}$ by

$$
\bar{T}((a, x))=(0, T(x)) .
$$


Then, straightforward calculations yield the following result.

Lemma 3.5. The operators $\bar{D}$ and $\bar{T}$ defined above are (continuous) derivations. Furthermore, the derivation $\bar{D}$ is inner if and only if $D$ is inner, and $\bar{T}$ is inner if and only if $T=0$.

\section{Proofs of the main theorems}

We first prove Theorem 2.1.

Proof. Denote by $\Delta_{1}$ the projection from $(\mathfrak{A} \oplus X)^{(2 m+1)}$ onto $\mathfrak{A}^{(2 m+1)}$ with kernel $X^{(2 m+1)}$. Let $\Delta_{2}$ be the projection $i d-\Delta_{1}:(\mathfrak{A} \oplus X)^{(2 m+1)} \rightarrow X^{(2 m+1)}$, and let $\tau_{1}$ : $\mathfrak{A} \rightarrow \mathfrak{A} \oplus X$ be the inclusion mapping (i.e., $\tau_{1}(a)=(a, 0)$ ). Then $\Delta_{1}$ and $\Delta_{2}$ are $\mathfrak{A}$-bimodule morphisms, and $\tau_{1}$ is an algebra homomorphism.

We now prove the sufficiency in Theorem 2.1. Suppose that conditions 1-4 hold. Suppose also that $D: \mathfrak{A} \oplus X \rightarrow(\mathfrak{A} \oplus X)^{(2 m+1)}$ is a continuous derivation. Then $D \circ \tau_{1}: \mathfrak{A} \rightarrow(\mathfrak{A} \oplus X)^{(2 m+1)}$ is a continuous derivation. This implies that $\Delta_{1} \circ D \circ \tau_{1}$ : $\mathfrak{A} \rightarrow \mathfrak{A}^{(2 m+1)}$ and $\Delta_{2} \circ D \circ \tau_{1}: \mathfrak{A} \rightarrow X^{(2 m+1)}$ are continuous derivations. By conditions 1 and 2, they are inner. Therefore, $D \circ \tau_{1}$ is inner. From Lemmas 3.5 and 3.4

$$
\overline{D \circ \tau_{1}}=\overline{\Delta_{1} \circ D \circ \tau_{1}}+\overline{\Delta_{2} \circ D \circ \tau_{1}}: \mathfrak{A} \oplus X \rightarrow(\mathfrak{A} \oplus X)^{(2 m+1)}
$$

is a continuous derivation, and there is a continuous derivation $\widetilde{D}: \mathfrak{A} \oplus X \rightarrow$ $(\mathfrak{A} \oplus X)^{(2 m+1)}$ satisfying $\widetilde{D}((a, 0))=0$ for $a \in \mathfrak{A}$ and such that $\overline{D \circ \tau_{1}}-\widetilde{D}$ is inner. On the other hand,

$$
\begin{aligned}
\left(D-\overline{D \circ \tau_{1}}\right)((a, 0)) & =D((a, 0))-\overline{D \circ \tau_{1}}((a, 0)) \\
& =D \circ \tau_{1}(a)-D \circ \tau_{1}(a)=0 \quad(a \in \mathfrak{A}) .
\end{aligned}
$$

Let $\widehat{D}=D-\overline{D \circ \tau_{1}}+\widetilde{D}$. Then $\widehat{D}$ is a continuous derivation from $\mathfrak{A} \oplus X$ into $(\mathfrak{A} \oplus X)^{(2 m+1)}$ satisfying $\widehat{D}((a, 0))=0$ for $a \in \mathfrak{A}$. Moreover,

$$
\widehat{D}((0, a x))=\widehat{D}((a, 0) \cdot(0, x))=(a, 0) \cdot \widehat{D}((0, x))=a \widehat{D}((0, x)) \quad(a \in \mathfrak{A}, x \in X),
$$

and

$$
\widehat{D}((0, x a))=\widehat{D}((0, x) \cdot(a, 0))=\widehat{D}((0, x)) a \quad(a \in \mathfrak{A}, x \in X) .
$$

Denote by $\tau_{2}: X \rightarrow \mathfrak{A} \oplus X$ the inclusion mapping given by $\tau_{2}(x)=(0, x)(x \in X)$. Then $\widehat{D} \circ \tau_{2}: X \rightarrow(\mathfrak{A} \oplus X)^{(2 m+1)}$ is a continuous $\mathfrak{A}$-bimodule morphism. From condition 3 , there exists $F \in X^{(2 m+1)}$ for which $\Delta_{1} \circ \widehat{D} \circ \tau_{2}(x)=x F-F x$, and $a F-F a=0$ for $x \in X$ and $a \in \mathfrak{A}$. Since

$$
\begin{aligned}
(0,0) & =\widehat{D}((0,0))=\widehat{D}((0, x) \cdot(0, y)) \\
& =\widehat{D}((0, x)) \cdot(0, y)+(0, x) \cdot \widehat{D}((0, y)) \\
& =\left(\left[\Delta_{2} \circ \widehat{D}((0, x))\right] y, 0\right)+\left(x\left[\Delta_{2} \circ \widehat{D}((0, y))\right], 0\right) \\
& =\left(\left[\Delta_{2} \circ \widehat{D} \circ \tau_{2}(x)\right] y+x\left[\Delta_{2} \circ \widehat{D} \circ \tau_{2}(y)\right], 0\right),
\end{aligned}
$$

we have

$$
\left(\Delta_{2} \circ \widehat{D} \circ \tau_{2}(x)\right) y+x\left(\Delta_{2} \circ \widehat{D} \circ \tau_{2}(y)\right)=0 \quad(x, y \in X) .
$$


From condition $4, \Delta_{2} \circ \widehat{D} \circ \tau_{2}=0$. Thus,

$$
\begin{aligned}
\widehat{D}((a, x)) & =\widehat{D}((0, x))=\widehat{D} \circ \tau_{2}(x) \\
& =\left(\Delta_{1} \circ \widehat{D} \circ \tau_{2}(x), \Delta_{2} \circ \widehat{D} \circ \tau_{2}(x)\right) \\
& =(x F-F x, 0)=(a, x) \cdot(0, F)-(0, F) \cdot(a, x) .
\end{aligned}
$$

We have that $\widehat{D}$ is inner. Thus $D=\widehat{D}+\left(\overline{D \circ \tau_{1}}-\widetilde{D}\right)$ is inner. This proves that $\mathfrak{A} \oplus X$ is $(2 m+1)$-weakly amenable.

Necessity: Suppose that $\mathfrak{A} \oplus X$ is $(2 m+1)$-weakly amenable. Then from Lemmas 3.5 and 3.4 $\mathcal{H}^{1}\left(\mathfrak{A}, \mathfrak{A}^{(2 m+1)}\right)=\{0\}$ and $\mathcal{H}^{1}\left(\mathfrak{A}, X^{(2 m+1)}\right)=\{0\}$. Therefore, conditions 1 and 2 hold. Furthermore, Lemma 3.1 gives condition 3, and Lemma 3.5 shows that condition 4 holds. This completes the proof of Theorem 2.1

We now turn to the proof of Theorem [2.2.

Proof. Denote by $\tau_{1}$ and $\tau_{2}$ the inclusion mappings described in the preceding proof from, respectively, $\mathfrak{A}$ and $X$ into $\mathfrak{A} \oplus X$, and denote by $\Delta_{1}$ and $\Delta_{2}$ the natural projections from $(\mathfrak{A} \oplus X)^{(2 m)}$ onto $\mathfrak{A}^{(2 m)}$ and $X^{(2 m)}$, respectively. These are $\mathfrak{A}$-bimodule morphisms.

To prove the sufficiency we assume that conditions 1-4 in Theorem 2.2 hold. Let $D:(\mathfrak{A} \oplus X) \rightarrow(\mathfrak{A} \oplus X)^{(2 m)}$ be a continuous derivation. Then $\Delta_{1} \circ D \circ \tau_{1}$ : $\mathfrak{A} \rightarrow \mathfrak{A}^{(2 m)}$ and $\Delta_{2} \circ D \circ \tau_{1}: \mathfrak{A} \rightarrow X^{(2 m)}$ are continuous derivations.

Claim 1: $\Delta_{1} \circ D \circ \tau_{2}: X \rightarrow \mathfrak{A}^{(2 m)}$ is trivial.

Let $\Gamma=\Delta_{1} \circ D \circ \tau_{2}$. To prove claim 1 , by condition 3 it suffices to show that $\Gamma$ is an $\mathfrak{A}$-bimodule morphism satisfying $x \Gamma(y)+\Gamma(x) y=0$ in $X^{(2 m)}$ for $x, y \in X$. In fact,

$$
\begin{aligned}
0=D((0,0)) & =D((0, x) \cdot(0, y))=D((0, x)) \cdot(0, y)+(0, x) \cdot D((0, y)) \\
& =(0, \Gamma(x) y)+(0, x \Gamma(y))
\end{aligned}
$$

Thus, $x \Gamma(y)+\Gamma(x) y=0$. On the other hand,

$$
\begin{aligned}
\Gamma(a x) & =\Delta_{1} \circ D((0, a x))=\Delta_{1} \circ D((a .0) \cdot(0, x)) \\
& =\Delta_{1}(D((a, 0)) \cdot(0, x)+(a, 0) \cdot D((0, x))) \\
& =\Delta_{1}((a, 0) \cdot D((0, x)))=\Delta_{1}\left(a D \circ \tau_{2}(x)\right)=a \Gamma(x) .
\end{aligned}
$$

Similarly, $\Gamma(x a)=\Gamma(x) a$ and so $\Gamma$ is an $\mathfrak{A}$-bimodule morphism. Therefore, claim 1 is true.

Now let $T=\Delta_{2} \circ D \circ \tau_{2}: X \rightarrow X^{(2 m)}$, and set $D_{1}=\Delta_{1} \circ D \circ \tau_{1}: \mathfrak{A} \rightarrow \mathfrak{A}^{(2 m)}$.

Claim 2: $T(a x)=D_{1}(a) x+a T(x)$ and $T(x a)=x D_{1}(a)+T(x) a$ for $a \in \mathfrak{A}$ and $x \in X$.

In fact, from claim 1 ,

$$
\begin{aligned}
(0, T(a x)) & =D((0, a x))=D((a, 0) \cdot(0, x))=D((a, 0)) \cdot(0, x)+(a, 0) \cdot D((0, x)) \\
& =\left(0, D_{1}(a) x\right)+a(0, T(x))=\left(0, D_{1}(a) x+a T(x)\right) .
\end{aligned}
$$

Similarly, $(0, T(x a))=\left(0, x D_{1}(a)+T(x) a\right)$, for $a \in \mathfrak{A}$ and $x \in X$. Thus, claim 2 is true.

Therefore, by condition $1, D_{1}=\Delta_{1} \circ D \circ \tau_{1}$ is inner. Suppose that $u \in \mathfrak{A}^{(2 m)}$ satisfies $D_{1}(a)=a u-u a$ for $a \in \mathfrak{A}$. Let $T_{1}: X \rightarrow X^{(2 m)}$ be defined by $T_{1}(x)=x u-u x$ 
for $x \in X$. Then $T-T_{1}: X \rightarrow X^{(2 m)}$ is a continuous $\mathfrak{A}$-bimodule morphism. In fact, from claim 2 , for $a \in \mathfrak{A}$ and $x \in X$,

$$
\begin{aligned}
\left(T-T_{1}\right)(a x) & =T(a x)-T_{1}(a x)=\left(D_{1}(a) x+a T(x)\right)-(a x u-u a x) \\
& =(a u-u a) x+a T(x)-(a x u-u a x) \\
& =a(u x-x u)+a T(x)=a\left(T-T_{1}\right)(x) .
\end{aligned}
$$

Similarly, $T-T_{1}$ is a right $\mathfrak{A}$-module morphism. From condition 4 , there is a $v \in \mathfrak{A}^{(2 m)}$ such that $a v=v a$ for $a \in \mathfrak{A}$, and $\left(T-T_{1}\right)(x)=x v-v x$ for $x \in X$. From Lemma 3.2, we have that

$$
\overline{T-T_{1}}:(a, x) \mapsto\left(0,\left(T-T_{1}\right)(x)\right), \quad \mathfrak{A} \oplus X \rightarrow(\mathfrak{A} \oplus X)^{(2 m)}
$$

is an inner derivation.

Since $\Delta_{2} \circ D \circ \tau_{1}: \mathfrak{A} \rightarrow X^{(2 m)}$ is a continuous derivation, it is inner by condition 2 . From Lemma 3.5,

$$
\overline{\Delta_{2} \circ D \circ \tau_{1}}:(a, x) \mapsto\left(0, \Delta_{2} \circ D \circ \tau_{1}(a)\right), \quad \mathfrak{A} \oplus X \rightarrow(\mathfrak{A} \oplus X)^{(2 m)}
$$

is also inner. Using claim 1, we now have

$$
\begin{aligned}
D((a, x)) & =\left(D_{1}(a), \quad \Delta_{2} \circ D \circ \tau_{1}(a)+T(x)\right) \\
& =\overline{\Delta_{2} \circ D \circ \tau_{1}}((a, x))+\left(\overline{T-T_{1}}\right)((a, x))+\left(D_{1}(a), T_{1}(x)\right) .
\end{aligned}
$$

Since

$$
\left(D_{1}(a), T_{1}(x)\right)=(a u-u a, x u-u x)=(a, x) \cdot(u, 0)-(u, 0) \cdot(a, x),
$$

for $a \in \mathfrak{A}$ and $x \in X$, it gives an inner derivation from $\mathfrak{A} \oplus X$ into $(\mathfrak{A} \oplus X)^{(2 m)}$. Hence as a sum of three inner derivations, $D$ is inner. This shows that under conditions $1-4$ of Theorem 2.2 $\mathfrak{A} \oplus X$ is $2 m$-weakly amenable.

Now we prove the necessity. Suppose that $\mathfrak{A} \oplus X$ is $2 m$-weakly amenable. Let $D: \mathfrak{A} \rightarrow \mathfrak{A}^{(2 m)}$ be a continuous derivation with the property given in condition 1 . Then $\bar{D}: \mathfrak{A} \oplus X \rightarrow(\mathfrak{A} \oplus X)^{(2 m)}$ defined by

$$
\bar{D}((a, x))=(D(a), T(x)),(a, x) \in \mathfrak{A} \oplus X,
$$

is a continuous derivation and hence is inner. This implies that $D$ is inner, and so condition 1 holds. The other conditions are consequences of Lemma 3.5 and Lemma 3.2

The proof is complete.

\section{The algebras $\mathfrak{A} \oplus \mathfrak{A}$ AND $\mathfrak{A} \oplus \mathfrak{A}^{*}$}

In this and the following section we consider several concrete cases. This section deals mainly with the two cases $X=\mathfrak{A}$ and $X=\mathfrak{A}^{*}$ as Banach $\mathfrak{A}$-bimodules.

We first note that, if $\mathfrak{A}$ is not amenable, then there is a Banach $\mathfrak{A}$-bimodule $X$ such that $\mathcal{H}^{1}\left(\mathfrak{A}, X^{*}\right) \neq\{0\}$. From Theorem 2.1, for this $X, \mathfrak{A} \oplus X$ is not weakly amenable. In fact, the Banach algebra $\mathfrak{A} \oplus X$ is never weakly amenable when $X=\mathfrak{A}^{*}$, as implied in the following proposition.

Proposition 5.1. Suppose that $\mathfrak{A}$ is a Banach algebra. Then $\mathfrak{A} \oplus \mathfrak{A}^{*}$ is not nweakly amenable for every $n \geq 0$. 
Proof. From Proposition 1.2 of 10, it suffices to prove the cases of $n=0, n=1$ and $n=2$. Note that condition $3^{0}$ does not hold, because the identity mapping from $X\left(=\mathfrak{A}^{*}\right)$ onto $\mathfrak{A}^{*}$ is a nonzero, continuous $\mathfrak{A}$-bimodule morphism. So the proposition is true for $n=1$.

For $n=2 m$ with $m=0$ or $m=1$, if condition 4 in Theorem 2.2 holds for $X=\mathfrak{A}^{*}$, then the operator $T$ described in this condition has the property that $T(f) \in \mathfrak{A}^{\perp}$ for $f \in X$. In fact, for $a \in \mathfrak{A}$, we have

$$
\langle a, T(f)\rangle=\langle a, f \cdot u-u \cdot f\rangle=\langle a f-f a, u\rangle=\langle f, u a-a u\rangle=0 .
$$

But $X=\mathfrak{A}^{*}$ certainly does not annihilate $\mathfrak{A}$. So, as $\mathfrak{A}$-bimodule morphisms, the identity mapping (in the case $m=0$ ) from $X$ onto $X$ and the inclusion mapping (in the case $m=1$ ) from $X$ into $X^{* *}$ do not satisfy condition 4 . Consequently, $\mathfrak{A} \oplus \mathfrak{A}^{*}$ is not $2 m$-weakly amenable for $m=0$ and 1 .

Now we consider the case that $X=\mathfrak{A}$. To avoid any confusion, from now on, when we regard $\mathfrak{A}$ as an $\mathfrak{A}$-bimodule, we will use the notation $A$ instead of $\mathfrak{A}$. If $X=A$, condition 4 in Theorem 2.2 never holds for any integer $m$ (the canonical embedding is a nonzero morphism). It turns out that $\mathfrak{A} \oplus A$ is never $2 m$-weakly amenable for any $m \geq 0$. If $\mathfrak{A}$ is commutative, for the same reason we can conclude more as in the next proposition. Recall that an $\mathfrak{A}$-bimodule $X$ is symmetric if $a x=x a$ for $a \in \mathfrak{A}$ and $x \in X$.

Proposition 5.2. Suppose that $\mathfrak{A}$ is a commutative Banach algebra. Then for every nonzero, symmetric $\mathfrak{A}$-bimodule $X, \mathfrak{A} \oplus X$ is not $2 m$-weakly amenable.

Proof. Let $X$ be symmetric. Then $x u=u x$ for $u \in \mathfrak{A}^{(2 m)}$ and $x \in X$. Since the canonical embedding from $X$ into $X^{(2 m)}$ is a nontrivial $\mathfrak{A}$-bimodule morphism, condition 4 in Theorem 2.2 does not hold for such a module $X$.

But $\mathfrak{A} \oplus A$ can be weakly amenable. Before giving an example let us go through some relation identities for corresponding elements of $A^{(n)}$ and $\mathfrak{A}^{(n)}$. Suppose that $\phi \in A^{(n)}$. We denote the same element in $\mathfrak{A}^{(n)}$ by $\tilde{\phi}$.

Lemma 5.3. Suppose that $\mathfrak{A}$ is a Banach algebra, and let $m \geq 0$. Then, for $\phi, \psi \in A^{(2 m)}$ and $F \in A^{(2 m+1)}$, we have

$$
(\tilde{\phi} \psi)^{\sim}=\tilde{\phi} \tilde{\psi}=(\phi \tilde{\psi})^{\sim}, \quad \phi F=(\tilde{\phi} F)^{\sim}=\tilde{\phi} \widetilde{F}, \quad F \phi=(F \tilde{\phi})^{\sim}=\tilde{F} \tilde{\phi} .
$$

Proof. It is straightforward to check the identities for the case $m=0$. Then, an induction on $m$ completes the proof for the general case.

A special case of Lemma 5.3 is the following group of identities which will be used in the proof of the next theorem:

$$
\begin{aligned}
(a \phi)^{\sim} & =a \tilde{\phi}, \quad(\phi a)^{\sim}=\tilde{\phi} a, \\
x F=(\tilde{x} F)^{\sim} & =\tilde{x} \widetilde{F}, \quad F x=(F \tilde{x})^{\sim}=\tilde{F} \tilde{x},
\end{aligned}
$$

where $a \in \mathfrak{A}, x \in A, \phi \in A^{(2 m)}$ and $F \in A^{(2 m+1)}$. From these identities, we also see that, for $X=A$ and $m \geq 0$, condition 3 in Theorem 2.1 holds if and only if there is no nonzero $\mathfrak{A}$-bimodule morphism $T$ from $A$ into $A^{(2 m+1)}$, and that, if this is the case, then condition 4 holds automatically. Moreover, with $X=A$, conditions 1 and 2 of Theorem 2.1] are the same. 
Theorem 5.4. For a Banach algebra $\mathfrak{A}$ :

1. if $\operatorname{span}\{a b-b a ; a, b \in \mathfrak{A}\}$ is not dense in $\mathfrak{A}$, then $\mathfrak{A} \oplus A$ is not weakly amenable;

2. if span $\{a b-b a ; a, b \in \mathfrak{A}\}$ is dense in $\mathfrak{A}$, then $\mathfrak{A} \oplus A$ is weakly amenable, provided that $\mathfrak{A}$ is weakly amenable and has a bounded approximate identity.

Proof. By condition 1 of Theorem 2.1 without loss of generality, we can assume that $\mathfrak{A}$ is weakly amenable for both cases. If $\operatorname{span}\{a b-b a ; a, b \in \mathfrak{A}\}$ is not dense in $\mathfrak{A}$, then there exists $f \in \mathfrak{A}^{*}$ such that $f \neq 0$ and $\langle a b-b a, f\rangle=0$ for $a, b \in \mathfrak{A}$. So $a f=f a$ for $a \in \mathfrak{A}$. Then $T: A \rightarrow \mathfrak{A}^{*}$, defined by

$$
T(x)=\tilde{x} f=f \tilde{x},
$$

is an $\mathfrak{A}$-bimodule morphism. According to Proposition 1.3 of [10], $\mathfrak{A}^{2}$, the linear span of all product elements $a b, a, b \in \mathfrak{A}$, is dense in $\mathfrak{A}$. So there are $a, b \in \mathfrak{A}$ such that $\langle a b, f\rangle \neq 0$. This implies that $T \neq 0$. Therefore, condition $3^{0}$ does not hold. As a consequence, $\mathfrak{A} \oplus A$ is not weakly amenable.

If $\operatorname{span}\{a b-b a ; a, b \in \mathfrak{A}\}$ is dense in $\mathfrak{A}$, and $\mathfrak{A}$ has a bounded approximate identity $\left(e_{i}\right)$, then, for every given continuous $\mathfrak{A}$-bimodule morphism $T: A \rightarrow \mathfrak{A}^{*}$, we have $T(a)=a f=f a$, where $f$ is a weak* cluster point of $\left(T\left(e_{i}\right)\right)$. Therefore, $\langle a b-b a, f\rangle=0$ for all $a, b \in \mathfrak{A}$. This shows that $f=0$ and hence $T=0$. Thus conditions 3 and 4 in Theorem 2.1 hold for $m=0$. The other two conditions hold automatically for $m=0$. So, from Theorem 2.1 the second statement of the theorem is true.

From case 1 of Theorem 5.4 we immediately have the following corollary.

Corollary 5.5. If $\mathfrak{A}$ is a commutative Banach algebra, then $\mathfrak{A} \oplus A$ is not weakly amenable.

Let $\mathcal{H}$ be an infinite-dimensional Hilbert space. According to a classical result due to Halmos (Theorem 8 of [18]), every element in $B(\mathcal{H})$ can be written as a sum of two commutators (see also [4] and [5]). Together with the fact that $B(\mathcal{H})$ has an identity and, as a $C^{*}$-algebra, is weakly amenable [17], from Theorem 5.4 we see that $B(\mathcal{H}) \oplus B(\mathcal{H})$ is weakly amenable. Later in this section we will see that it is in fact $(2 m+1)$-weakly amenable.

Proposition 5.6. Suppose that $V=\operatorname{span}\left\{a u-u a ; u \in \mathfrak{A}^{* *}, a \in \mathfrak{A}\right\}$ is not dense in $\mathfrak{A A}^{* *}+\mathfrak{A}^{* *} \mathfrak{A}$ (if $\mathfrak{A}$ has an identity, this is equivalent to saying that $V$ is not dense in $\left.\mathfrak{A}^{* *}\right)$. Then $\mathfrak{A} \oplus A$ is not 3 -weakly amenable.

Proof. In fact, in this case $\mathfrak{A}^{* *} \mathfrak{A} \nsubseteq c l(V)$, since otherwise it would follow that both $\mathfrak{A A}^{* *}$ and $\mathfrak{A}^{* *} \mathfrak{A}$ were in $\operatorname{cl}(V)$, and then $\operatorname{cl}(V) \supseteq \mathfrak{A A}^{* *}+\mathfrak{A}^{* *} \mathfrak{A}$, which contradicts the assumption that $V$ is not dense in $\mathfrak{A A}^{* *}+\mathfrak{A}^{* *} \mathfrak{A}$.

Hence, from the Hahn-Banach Theorem, there exists $F \in \mathfrak{A}^{* * *}$ such that $\left.F\right|_{V}=$ 0 , but $F \neq 0$ on $\mathfrak{A}^{* *} \mathfrak{A}$. This implies that $a F=F a$ for all $a \in \mathfrak{A}$ and $a F \neq 0$ for some $a \in \mathfrak{A}$. Define $T: A \rightarrow \mathfrak{A}^{* * *}$ by $T(x)=\tilde{x} F(=F \tilde{x})$. Then, $T$ is a nonzero, continuous $\mathfrak{A}$-bimodule morphism from $A$ into $\mathfrak{A}^{* * *}$. Therefore, condition 3 in Theorem 2.1 does not hold for $m=1$. This shows that $\mathfrak{A} \oplus A$ is not 3-weakly amenable.

Regarding the open question of whether weak amenability implies 3-weak amenability, Theorem 5.4 and Proposition [5.6] suggest that one might find a counterexample in the Banach algebras of the form $\mathfrak{A} \oplus A$. Unfortunately, $B(\mathcal{H})$ cannot be 
a candidate. We can see this from the next two lemmas. The following lemma is basically Theorem 8 in [18], but we have highlighted some of its features which will be useful for our purposes.

Lemma 5.7. Suppose that $\mathcal{H}$ is an infinite-dimensional Hilbert space. Then there are two elements $Q_{0}$ and $S_{0}$ in $B(\mathcal{H})$ such that, for each $B \in B(\mathcal{H})$, there exist $P_{B} \in B(\mathcal{H})$ and $R_{B} \in B(\mathcal{H})$ with $\left\|P_{B}\right\| \leq\|B\|$ and $\left\|R_{B}\right\| \leq\|B\|$ for which

$$
B=\left(P_{B} \circ Q_{0}-Q_{0} \circ P_{B}\right)+\left(R_{B} \circ S_{0}-S_{0} \circ R_{B}\right) \text {. }
$$

Proof. For an infinite-dimensional Hilbert space $\mathcal{H}$, there exists an isometry $\eta$ : $\mathcal{H} \rightarrow \sum_{i=1}^{\infty} \dot{+} \mathcal{H}_{i}$, where $\sum_{i=1}^{\infty} \dot{+}$ denotes an $l_{2}$ direct sum and each $\mathcal{H}_{i}$ is a copy of $\mathcal{H}$.

Let $Q: \mathcal{H} \rightarrow \sum_{i=1}^{\infty} \dot{+} \mathcal{H}_{i}$ and $S: \sum_{i=1}^{\infty} \dot{+} \mathcal{H}_{i} \rightarrow \sum_{i=1}^{\infty} \dot{+} \mathcal{H}_{i}$ be the bounded operators given by the infinite matrices

$$
Q=\left(\begin{array}{c}
I \\
0 \\
0 \\
\vdots
\end{array}\right) \quad \text { and } S=\left(\begin{array}{ccccc}
0 & 0 & 0 & 0 & \cdots \\
I & 0 & 0 & 0 & \cdots \\
0 & I & 0 & 0 & \ldots \\
0 & 0 & I & 0 & \ldots \\
\vdots & \vdots & 0 & \ddots &
\end{array}\right)
$$

Let $Q_{0}=\eta^{-1} \circ Q$ and $S_{0}=\eta^{-1} \circ S \circ \eta$. Then $Q_{0}, S_{0} \in B(\mathcal{H})$. Given an element $B \in B(\mathcal{H})$, let $P: \sum_{i=1}^{\infty} \dot{+} \mathcal{H}_{i} \rightarrow \mathcal{H}$ and $R: \sum_{i=1}^{\infty} \dot{+} \mathcal{H}_{i} \rightarrow \sum_{i=1}^{\infty} \dot{+} \mathcal{H}_{i}$ be the bounded operators given by the infinite matrices

$$
P=\left(\begin{array}{llll}
B & 0 & 0 & \cdots
\end{array}\right) \quad \text { and } R=\left(\begin{array}{ccccc}
0 & B & 0 & 0 & \cdots \\
0 & 0 & B & 0 & \cdots \\
0 & 0 & 0 & B & \cdots \\
\vdots & \vdots & \vdots & 0 & \ddots
\end{array}\right) \text {. }
$$

Take $P_{B}=P \circ \eta$ and $R_{B}=\eta^{-1} \circ R \circ \eta$. Then $P_{B}, R_{B} \in B(\mathcal{H})$ and $\left\|P_{B}\right\| \leq\|B\|$, $\left\|R_{B}\right\| \leq\|B\|$. We have that $B=\left(P_{B} \circ Q_{0}-Q_{0} \circ P_{B}\right)+\left(R_{B} \circ S_{0}-S_{0} \circ R_{B}\right)$.

The following result on the $2 n$-th dual of $B(\mathcal{H})$ seems not to be known.

Lemma 5.8. For every integer $n \geq 0$,

$$
B(\mathcal{H})^{(2 n)}=\operatorname{span}\left\{a u-u a ; a \in B(\mathcal{H}), u \in B(\mathcal{H})^{(2 n)}\right\}
$$

Proof. By taking weak* limits and using induction, one can show the result immediately from Lemma 5.7 .

Proposition 5.9. For each integer $m \geq 0, B(\mathcal{H}) \oplus B(\mathcal{H})$ is $(2 m+1)$-weakly amenable but is not $2 m$-weakly amenable.

Proof. First, as a $C^{*}$-algebra, $B(\mathcal{H})$ is permanently weakly amenable. So conditions 1 and 2 of Theorem 2.1 hold for $X=\mathfrak{A}=B(\mathcal{H})$ and $m \geq 0$. To show that conditions 3 and 4 also hold, it suffices to prove that every continuous $B(\mathcal{H})$ bimodule morphism $T$ from $B(\mathcal{H})$ into $B(\mathcal{H})^{(2 m+1)}$ is trivial.

In fact, letting $e$ be the identity of $B(\mathcal{H})$ and $F=T(e)$, we have $T(a)=a F=F a$ for all $a \in B(\mathcal{H})$. Therefore, for all $u \in B(\mathcal{H})^{(2 m)}$, we have $\langle a u-u a, F\rangle=0$. From Lemma 5.8 this implies that $F=0$. Hence $T=0$. Therefore, $B(\mathcal{H}) \oplus B(\mathcal{H})$ is $(2 m+1)$-weakly amenable for $m \geq 0$. 
On the other hand, we have indicated in the paragraph before Proposition $[5.2$ that $\mathfrak{A} \oplus A$ is never $2 m$-weakly amenable. So $B(\mathcal{H}) \oplus B(\mathcal{H})$ is not $2 m$-weakly amenable for $m \geq 0$. This completes the proof.

Remark 5.10. Denote by $K(\mathcal{H})$ the algebra of compact operators on $\mathcal{H}$. Using Theorem 1 of [29] one can also prove that $K(\mathcal{H}) \oplus K(\mathcal{H})$ and $B(\mathcal{H}) \oplus K(\mathcal{H})$ are $(2 m+1)$ - (but not $2 m-)$ weakly amenable. On the other hand, it is interesting to recall Proposition 2.4 which implies that $K(\mathcal{H}) \oplus B(\mathcal{H})$ is not weakly amenable.

\section{The algebra $\mathfrak{A} \oplus X_{0}$}

In this section we consider the case that the module action on one side of $X$ is trivial. We denote by $X_{0}$ (respectively, ${ }_{0} Y$ ) specifically the $\mathfrak{A}$-bimodules with right (respectively, left) module action trivial. We observe that, when $X=X_{0}$, conditions 3 and 4 in Theorem 2.1 are reduced, respectively, to the following:

$3_{0}^{\prime}$. for each continuous $\mathfrak{A}$-bimodule morphism $\Gamma: X_{0} \rightarrow \mathfrak{A}^{(2 m+1)}$, there is $F \in$ $X_{0}^{(2 m+1)}$ such that $F a=0$ for $a \in \mathfrak{A}$ and $\Gamma(x)=x F$ for $x \in X_{0}$;

$4_{0}^{\prime} . \mathfrak{A} X_{0}$ is dense in $X_{0}$.

Also, conditions 1, 3 and 4 in Theorem 2.2 are reduced, respectively, to the following:

$1_{0}^{\prime \prime}$. every continuous derivation $D: \mathfrak{A} \rightarrow \mathfrak{A}^{(2 m)}$ with the property that there is a continuous operator $T: X_{0} \rightarrow X_{0}^{(2 m)}$ such that $T(a x)=D(a) x+a T(x)$ for $a \in \mathfrak{A}$ and $x \in X_{0}$ is inner;

$3_{0}^{\prime \prime}$. the only continuous $\mathfrak{A}$-bimodule morphism $\Gamma: X_{0} \rightarrow \mathfrak{A}^{(2 m)}$ satisfying $\Gamma(x) y=$ $0\left(x, y \in X_{0}\right)$ in $X_{0}^{(2 m)}$ is zero;

$4_{0}^{\prime \prime}$. for every continuous $\mathfrak{A}$-bimodule morphism $T: X_{0} \rightarrow X_{0}^{(2 m)}$, there exists $u \in \mathfrak{A}^{(2 m)}$ such that $a u=u a$ for $a \in \mathfrak{A}$ and $T(x)=u x$ for $x \in X_{0}$.

Proposition 6.1. Suppose that $\mathfrak{A}$ is a $(2 m+1)$-weakly amenable Banach algebra with a bounded approximate identity and satisfying that $\mathfrak{A A}^{(2 m)}=\mathfrak{A}^{(2 m)}$. Then, $\mathfrak{A} \oplus X_{0}$ is $(2 m+1)$-weakly amenable if and only if $\mathfrak{A} X_{0}$ is dense in $X_{0}$.

Proof. Since $\mathfrak{A}$ has a bounded approximate identity, from Proposition 1.5 of [23], condition 2 in Theorem 2.1 always holds for $X=X_{0}$. If $\mathfrak{A A}^{(2 m)}=\mathfrak{A}^{(2 m)}$, then there is no nonzero, continuous $\mathfrak{A}$-bimodule morphism $T: X_{0} \rightarrow \mathfrak{A}^{(2 m+1)}$, since such a morphism must satisfy $\langle a u, T(x)\rangle=\langle u, T(x a)\rangle=0\left(a \in \mathfrak{A}, u \in \mathfrak{A}^{(2 m)}\right)$. So condition $3_{0}^{\prime}$ holds automatically.

For $m=0$, the above proposition yields the following.

Corollary 6.2. Suppose that $\mathfrak{A}$ is a weakly amenable Banach algebra with a bounded approximate identity. Then $\mathfrak{A} \oplus X_{0}$ is weakly amenable if and only if $\mathfrak{A} X_{0}$ is dense in $X_{0}$.

A dual result to Corollary 6.2 is as follows.

Corollary 6.3. Suppose that $\mathfrak{A}$ is a weakly amenable Banach algebra with a bounded approximate identity. Let ${ }_{0} Y$ be a Banach $\mathfrak{A}$-bimodule with left module action trivial. Then, $\mathfrak{A} \oplus_{0} Y$ is weakly amenable if and only if ${ }_{0} Y \mathfrak{A}$ is dense in ${ }_{0} Y$.

View $\mathfrak{A}$ as a left $\mathfrak{A}$-module and then impose a trivial right $\mathfrak{A}$-module action on it. This results in a Banach $\mathfrak{A}$-bimodule. We denote it by $A_{0}$. Suppose that $\phi \in A_{0}^{(n)}$. 
We denote the same element in $\mathfrak{A}^{(n)}$ by $\tilde{\phi}$. Similarly to Lemma 5.3, one can check that the following equalities hold:

$$
\begin{aligned}
& (u \phi)^{\sim}=u \tilde{\phi}, \quad \phi u=0, \quad \phi F=\tilde{\phi} \widetilde{F}, \\
& F \phi=0, \quad u F=0, \quad(F u)^{\sim}=\widetilde{F} u,
\end{aligned}
$$

where $u \in \mathfrak{A}^{(2 m)}, \phi \in A_{0}^{(2 m)}, F \in A_{0}^{(2 m+1)}(m \geq 0)$.

Proposition 6.4. Suppose that $\mathfrak{A}$ is a $(2 m+1)$-weakly amenable Banach algebra with a bounded approximate identity. Then $\mathfrak{A} \oplus A_{0}$ is $(2 m+1)$-weakly amenable.

Proof. As in the proof of Proposition 6.1] it suffices to verify conditions $3^{\prime}$ and $4^{\prime}$. Condition $4_{0}^{\prime}$ holds since $\mathfrak{A}$ has a left bounded approximate identity for $A_{0}$. Let $\left(x_{\alpha}\right) \subset A_{0}$ be a net such that $\left(\tilde{x}_{\alpha}\right)$ is a bounded approximate identity for $\mathfrak{A}$. If $\Gamma$ : $A_{0} \rightarrow \mathfrak{A}^{(2 m+1)}$ is a continuous $\mathfrak{A}$-bimodule morphism, we let $\widetilde{F}$ be a weak* ${ }^{*}$ cluster point of $\left(\Gamma\left(x_{\alpha}\right)\right)$. Let the element in $A_{0}^{(2 m+1)}$ corresponding to $\widetilde{F}$ be $F$. Then $F$ satisfies the requirement in condition $3_{0}^{\prime}$.

Concerning $2 m$-weak amenability, we have the following.

Proposition 6.5. Let $m \geq 1$, and suppose that $\mathfrak{A}$ is a commutative $2 m$-weakly amenable Banach algebra with a bounded approximate identity. Then $\mathfrak{A} \oplus A_{0}$ is $2 m$-weakly amenable.

Proof. It suffices to show that conditions $3_{0}^{\prime \prime}$ and $4_{0}^{\prime \prime}$ hold. Suppose that an $\mathfrak{A}-$ bimodule morphism $\Gamma: A_{0} \rightarrow \mathfrak{A}^{(2 m)}$ satisfies $\Gamma(x) y=0$ in $A_{0}^{(2 m)}\left(x, y \in A_{0}\right)$. Then

$$
0=(\Gamma(x) y)^{\sim}=\Gamma(x) \tilde{y}=\tilde{y} \Gamma(x)=\Gamma(\tilde{y} x) \quad\left(x, y \in A_{0}\right) .
$$

This implies that $\Gamma(a x)=0$ for $a \in \mathfrak{A}$ and $x \in A_{0}$. So $\Gamma(x)=0$ for all $x \in A_{0}$. Therefore, condition $3_{0}^{\prime \prime}$ holds.

Assume that $T: A_{0} \rightarrow A_{0}^{(2 m)}$ is a continuous $\mathfrak{A}$-bimodule morphism. Let $v$ be a weak* cluster point of $\left(T\left(x_{i}\right)\right)$, where $\left(\tilde{x}_{i}\right)$ is a bounded approximate identity for $\mathfrak{A}$. Let $u=\tilde{v}$. Then, $T(x)=\lim T\left(\tilde{x} x_{i}\right)=\tilde{x} v$. However, $(\tilde{x} v)^{\sim}=\tilde{x} \tilde{v}=\tilde{x} u=u \tilde{x}=$ $(u x)^{\sim}$. Hence $T(x)=u x$. On the other hand, $u a=a u$ since $\mathfrak{A}$ is commutative. Condition $4_{0}^{\prime \prime}$ holds.

Although we have already had an example of a Banach algebra which is $(2 m+1)$ weakly amenable but not $2 m$-weakly amenable (see Proposition 5.9; another known example is the nuclear algebra $\mathcal{N}(E)$ with $E$ a reflexive Banach space having the approximation property [10. Corollary 5.4]), we end this section by giving one more example of a weakly amenable Banach algebra which is not 2-weakly amenable.

Suppose that $\mathfrak{A}$ is a weakly amenable Banach algebra with a bounded approximate identity and satisfying that $\mathfrak{A A}^{*} \neq \mathfrak{A}^{*} \mathfrak{A}$ (an example is $\mathfrak{A}=L^{1}(G)$ with $G$ a non-SIN locally compact group; see [28] and [25] for the reference of SIN groups, and Theorem 32.44 of [20] as well as [26] for the property we need here). Without loss of generality, we assume that $\mathfrak{A R}^{*} \nsubseteq \mathfrak{A}^{*} \mathfrak{A}$.

Example 6.6. For the above Banach algebra $\mathfrak{A}, \mathfrak{A} \oplus A_{0}$ is weakly amenable but is not 2-weakly amenable. 
Proof. From Proposition [6.4, $\mathfrak{A} \oplus A_{0}$ is weakly amenable. We show that condition $3_{0}^{\prime \prime}$ does not hold for $m=1$. Take a $\phi \in \mathfrak{A}^{* *}$ for which $\left.\phi\right|_{\mathfrak{A}^{*}}=0$ but $\left.\phi\right|_{\mathfrak{A}^{*} \mathfrak{A}} \neq 0$ (notice that by Cohen's factorization theorem, $\mathfrak{A A}^{*}$ is closed in $\mathfrak{A}^{*}$ ). Then $\phi a=$ 0 for all $a \in \mathfrak{A}$ and $a \phi \neq 0$ for some $a \in \mathfrak{A}$. Let $T: A_{0} \rightarrow \mathfrak{A}^{* *}$ be defined by $T(x)=\tilde{x} \phi$. Then $T$ is a continuous $\mathfrak{A}$-bimodule morphism and $T \neq 0$. Since

$$
(T(x) y)^{\sim}=T(x) \tilde{y}=(\tilde{x} \phi) \tilde{y}=\tilde{x}(\phi \tilde{y})=0,
$$

we have $T(x) y=0$ for all $x, y \in A_{0}$. Therefore, condition $3_{0}^{\prime \prime}$ is not satisfied.

\section{WeAK AMENABILITY DOES NOT IMPLy 3-WEAK AMENABILITY}

Suppose that $X_{1}$ and $X_{2}$ are two Banach $\mathfrak{A}$-bimodules. We denote by $X_{1} \dot{+} X_{2}$ the direct module sum of $X_{1}$ and $X_{2}$, i.e., the $l_{1}$ direct sum of $X_{1}$ and $X_{2}$ with the module actions given by $a\left(x_{1}, x_{2}\right)=\left(a x_{1}, a x_{2}\right),\left(x_{1}, x_{2}\right) a=\left(x_{1} a, x_{2} a\right)$. For this module we have the following equality:

$$
\left(x_{1}, x_{2}\right) \cdot\left(f_{1}^{*}, f_{2}^{*}\right)=x_{1} f_{1}^{*}+x_{2} f_{2}^{*} \quad\left(\left(x_{1}, x_{2}\right) \in X_{1} \dot{+} X_{2},\left(f_{1}^{*}, f_{2}^{*}\right) \in\left(X_{1} \dot{+} X_{2}\right)^{*}\right) .
$$

In this section we shall first study the weak amenability of the Banach algebra $\mathfrak{A} \oplus\left(X_{1} \dot{+} X_{2}\right)$. Then we shall give an example of a weakly amenable Banach algebra of this form which is not 3-weakly amenable.

Lemma 7.1. Suppose that $\mathfrak{A} \oplus X_{1}$ and $\mathfrak{A} \oplus X_{2}$ are weakly amenable. Then the following are equivalent:

(i) $\mathfrak{A} \oplus\left(X_{1} \dot{+} X_{2}\right)$ is weakly amenable;

(ii) there is no nonzero, continuous $\mathfrak{A}$-bimodule morphism $\gamma: X_{1} \rightarrow X_{2}^{*}$;

(iii) there is no nonzero, continuous $\mathfrak{A}$-bimodule morphism $\eta: X_{2} \rightarrow X_{1}^{*}$.

Proof. Suppose that (i) holds. We show that (ii) also holds. Indeed, suppose that $\gamma: X_{1} \rightarrow X_{2}^{*}$ is a continuous $\mathfrak{A}$-bimodule morphism. Consider the continuous A-bimodule morphism $T: X_{1} \dot{+} X_{2} \rightarrow\left(X_{1} \dot{+} X_{2}\right)^{*}$ defined by

$$
T\left(\left(x_{1}, x_{2}\right)\right)=\left(-\gamma^{*}\left(x_{2}\right), \gamma\left(x_{1}\right)\right), \quad\left(x_{1}, x_{2}\right) \in X_{1} \dot{+} X_{2} .
$$

For $\left(x_{1}, x_{2}\right),\left(y_{1}, y_{2}\right) \in X_{1} \dot{+} X_{2}$, and $a \in \mathfrak{A}$, we have

$$
\begin{aligned}
& \left\langle a,\left(x_{1}, x_{2}\right) \cdot T\left(\left(y_{1}, y_{2}\right)\right)+T\left(\left(x_{1}, x_{2}\right)\right) \cdot\left(y_{1}, y_{2}\right)\right\rangle \\
& =\left\langle a,-x_{1} \gamma^{*}\left(y_{2}\right)+x_{2} \gamma\left(y_{1}\right)\right\rangle+\left\langle a,-\gamma^{*}\left(x_{2}\right) y_{1}+\gamma\left(x_{1}\right) y_{2}\right\rangle \\
& =\left\langle a,-\gamma\left(x_{1}\right) y_{2}+x_{2} \gamma\left(y_{1}\right)\right\rangle+\left\langle a,-x_{2} \gamma\left(y_{1}\right)+\gamma\left(x_{1}\right) y_{2}\right\rangle=0 .
\end{aligned}
$$

So $\left(x_{1}, x_{2}\right) \cdot T\left(\left(y_{1}, y_{2}\right)\right)+T\left(\left(x_{1}, x_{2}\right)\right) \cdot\left(y_{1}, y_{2}\right)=0$. Then, from condition 4 of Theorem 2.1, $T=0$. Thus $\gamma=0$. As a consequence, (ii) holds.

To prove that (ii) implies (iii), we suppose that $\eta: X_{2} \rightarrow X_{1}^{*}$ is a continuous A-bimodule morphism. Then $\gamma: X_{1} \rightarrow X_{2}^{*}$ defined by $\gamma=\left.\eta^{*}\right|_{X_{1}}$ is a continuous A-bimodule morphism. Therefore, $\gamma=0$. This implies that $\eta^{*}=0$ since $\eta^{*}$ is weak*-weak* continuous and $X_{1}$ is weak ${ }^{*}$ dense in $X_{1}^{* *}$. Thus, $\eta=0$, showing that (iii) holds. Similarly, one can prove that (iii) implies (ii).

Finally, we prove that (ii) + (iii) implies (i). Because $\mathfrak{A} \oplus X_{1}$ and $\mathfrak{A} \oplus X_{2}$ are weakly amenable, conditions $1-3$ of Theorem 2.1 hold automatically for $X=$ $X_{1} \dot{+} X_{2}$ and $m=0$. We show that condition 4 also holds. Suppose that $T: X \rightarrow X^{*}$ is a continuous $\mathfrak{A}$-bimodule morphism satisfying

$$
\left(x_{1}, x_{2}\right) \cdot T\left(\left(y_{1}, y_{2}\right)\right)+T\left(\left(x_{1}, x_{2}\right)\right) \cdot\left(y_{1}, y_{2}\right)=0 \quad\left(\left(x_{1}, x_{2}\right),\left(y_{1}, y_{2}\right) \in X\right) .
$$


Let $P_{i}: X^{*} \rightarrow X_{i}^{*}$ be the natural projections and let $\tau_{i}: X_{i} \rightarrow X$ be the natural embeddings, $i=1,2$. Then, by taking $x_{2}=y_{2}=0$ and $x_{1}=y_{1}=0$ separately, we have

$$
\begin{aligned}
& x_{1} \cdot P_{1} \circ T \circ \tau_{1}\left(y_{1}\right)+P_{1} \circ T \circ \tau_{1}\left(x_{1}\right) \cdot y_{1}=0, \\
& x_{2} \cdot P_{2} \circ T \circ \tau_{2}\left(y_{2}\right)+P_{2} \circ T \circ \tau_{2}\left(x_{2}\right) \cdot y_{2}=0,
\end{aligned}
$$

for all $x_{i}, y_{i} \in X_{i}, i=1,2$. So we have $P_{i} \circ T \circ \tau_{i}=0$ by applying condition 4 of Theorem 2.1 to the weakly amenable Banach algebras $\mathfrak{A} \oplus X_{i}, i=1,2$. Furthermore, (ii) and (iii) imply that $P_{1} \circ T \circ \tau_{2}: X_{2} \rightarrow X_{1}^{*}$ and $P_{2} \circ T \circ \tau_{1}: X_{1} \rightarrow X_{2}^{*}$ are trivial. Therefore, we have $T=0$. Condition 4 of Theorem 2.1 holds for $X=X_{1} \dot{+} X_{2}$. From Theorem 2.1, $\mathfrak{A} \oplus\left(X_{1} \dot{+} X_{2}\right)$ is weakly amenable. This completes the proof.

Proposition 7.2. The algebra $\mathfrak{A} \oplus\left(X_{1} \dot{+} X_{2}\right)$ is weakly amenable if and only if both $\mathfrak{A} \oplus X_{1}$ and $\mathfrak{A} \oplus X_{2}$ are weakly amenable and condition (ii) or condition (iii) in Lemma 7.1 holds.

Proof. If $\mathfrak{A} \oplus\left(X_{1} \dot{+} X_{2}\right)$ is weakly amenable, then conditions $1-4$ of Theorem 2.1 hold for this algebra. It follows that these conditions also hold for the algebras $\mathfrak{A} \oplus X_{1}$ and $\mathfrak{A} \oplus X_{2}$. So the latter two are also weakly amenable. The rest has been given in Lemma 7.1

In the remainder of the paper we focus on constructing an example of a weakly amenable Banach algebra which is not 3-weakly amenable. Recall that we always equip $\mathfrak{A}^{(2 m)}$ with the first Arens product. The following lemma has been proved in [31.

Lemma 7.3. Suppose that $\mathfrak{A}$ is a left (right) ideal in $\mathfrak{A}^{* *}$. Then it is also a left (respectively, right) ideal in $\mathfrak{A}^{(2 m)}$ for all $m \geq 1$.

Suppose that $\mathfrak{B}$ is a Banach algebra and $\mathfrak{A}=\mathfrak{B}^{* *}$. If $\mathfrak{B}$ is an ideal in $\mathfrak{B}^{* *}$, then a natural way to make $\mathfrak{B}$ an $\mathfrak{A}$-bimodule is using (the first) Arens product to give the module actions. In this way $\mathfrak{B}^{* *}$ is an $\mathfrak{A}^{* *}$-bimodule. For $b \in \mathfrak{B} \subset \mathfrak{B}^{* *}$ and $u \in \mathfrak{A}^{* *}$, the module coupling $u \cdot b$ and $b \cdot u$ result in elements of $\mathfrak{B}^{* *}$. Since $\mathfrak{B} \subset \mathfrak{B}^{(4)}\left(=\mathfrak{A}^{* *}\right)$, we can also consider the products $u b$ and $b u$ in the sense of Arens in $\mathfrak{B}^{(4)}$. But, from the above lemma, $u b, b u \in \mathfrak{B} \subset \mathfrak{B}^{* *}$. It is routine to check that, as elements in $\mathfrak{B}^{* *}, u \cdot b=u b$ and $b \cdot u=b u$.

From this point on, $\mathcal{H}$ will denote an infinite-dimensional, separable Hilbert space, $B(\mathcal{H})$ will denote the Banach algebra of all bounded operators on $\mathcal{H}$, and $K(\mathcal{H})$ the ideal of all compact operators on $\mathcal{H}$. It is well known that, with any Arens product, $K(\mathcal{H})^{* *}=B(\mathcal{H})$ (see [27, p. 103] for details).

Lemma 7.4. There is an element $a_{0} \in B(\mathcal{H})$ such that $a_{0} \notin K(\mathcal{H}), a_{0}$ is not right invertible in $B(\mathcal{H})$ and $a_{0} K(\mathcal{H})$ is dense in $K(\mathcal{H})$.

Proof. Let $\left(e_{i}\right)_{i=1}^{\infty}$ be an orthonormal basis of $\mathcal{H}$. Let $a_{0} \in B(\mathcal{H})$ be defined by

$$
a_{0}\left(e_{i}\right)= \begin{cases}\frac{1}{i} e_{i} & \text { if } i \text { is even } \\ e_{i} & \text { if } i \text { is odd. }\end{cases}
$$

Clearly, $a_{0} \notin K(\mathcal{H})$. Also, $a_{0}$ is neither right nor left invertible because any onesided inverse of $a_{0}$ must satisfy

$$
a_{0}^{-1}\left(e_{i}\right)= \begin{cases}i e_{i} & \text { if } i \text { is even } \\ e_{i} & \text { if } i \text { is odd }\end{cases}
$$


which cannot be a bounded operator.

For each $n \geq 1$, denote by $V_{n}$ the subspace of $\mathcal{H}$ generated by $\left\{e_{1}, e_{2}, \ldots, e_{n}\right\}$, and let $P_{n}$ be the orthogonal projection from $\mathcal{H}$ onto $V_{n}$. Then, from Corollary II.4.5 of [6], for every $k \in K(\mathcal{H})$ and $\varepsilon>0$, there is $n=n(k, \varepsilon)$, such that $\left\|P_{n} \circ k-k\right\|<\varepsilon$. For this $n=n(k, \varepsilon)$, let $b_{n} \in B(\mathcal{H})$ be defined by

$$
b_{n}\left(e_{i}\right)= \begin{cases}i e_{i} & \text { if } i \leq n \text { and } i \text { is even } \\ e_{i} & \text { if } i \leq n \text { and } i \text { is odd } \\ 0 & \text { for } i \geq n\end{cases}
$$

Then $a_{0} \circ b_{n}=P_{n}$ and $a_{0} \circ b_{n} \circ P_{n}=P_{n}^{2}=P_{n}$. Let $k_{n}=b_{n} \circ P_{n} \circ k$. Then $k_{n} \in K(\mathcal{H})$, and $a_{0} \circ k_{n}=P_{n} \circ k$. Also, $\left\|a_{0} \circ k_{n}-k\right\|=\left\|P_{n} \circ k-k\right\|<\varepsilon$. Since $k \in K(\mathcal{H})$ and $\varepsilon \geq 0$ are arbitrarily given, this shows that $a_{0} K(\mathcal{H})$ is dense in $K(\mathcal{H})$.

For the element $a_{0}$ in the above lemma, $a_{0} B(\mathcal{H})$ is a proper right ideal of $B(\mathcal{H})$ since the identity $1 \notin a_{0} B(\mathcal{H})$. The closure of $a_{0} B(\mathcal{H})$ is also a proper right ideal of $B(\mathcal{H})\left(\left[3\right.\right.$ p. 46]). So there is $F \in B(\mathcal{H})^{*}$ such that $F \neq 0$ but $F a_{0}=0$. Then, $F B(\mathcal{H}) \neq\{0\}$ is a right $B(\mathcal{H})$-submodule of $B(\mathcal{H})^{*}$. Take

$$
X_{0}=(K(\mathcal{H}))_{0} \text {, and }{ }_{0} Y={ }_{0}(\operatorname{cl}(F B(\mathcal{H})) .
$$

Then we have the following example.

Example 7.5. $B(\mathcal{H}) \oplus\left(X_{0} \dot{+}{ }_{0} Y\right)$ is weakly amenable but not 3-weakly amenable.

Proof. Clearly, we have $B(\mathcal{H}) X_{0}=X_{0}$ and ${ }_{0} Y B(\mathcal{H})={ }_{0} Y$. By Corollaries 6.2 and 6.3, the Banach algebras $B(\mathcal{H}) \oplus X_{0}$ and $B(\mathcal{H}) \oplus{ }_{0} Y$ are weakly amenable.

Suppose that $T:{ }_{0} Y \rightarrow X_{0}^{*}$ is a continuous $B(\mathcal{H})$-bimodule morphism. We prove that $T$ is trivial. Let $f=T(F)$. Then $f a_{0}=T\left(F a_{0}\right)=0$, and so $\left\langle a_{0} K(\mathcal{H}), f\right\rangle=$ $\{0\}$. We then have $f=0$ since $a_{0} K(\mathcal{H})$ is dense in $K(\mathcal{H})$. This shows that $T(F)=0$ and hence $T(F B(\mathcal{H}))=\{0\}$. Thus, $T=0$. From Proposition [7.2, $B(\mathcal{H}) \oplus\left(X_{0} \dot{+}{ }_{0} Y\right)$ is weakly amenable.

To prove that $B(\mathcal{H}) \oplus\left(X_{0} \dot{+}{ }_{0} Y\right)$ is not 3-weakly amenable, we show that it fails condition 4 of Theorem 2.1 for $m=1$. Since

$$
\left(X_{0}\right)^{* * *}={ }_{0}\left(K(\mathcal{H})^{* * *}\right)={ }_{0}\left(B(\mathcal{H})^{*}\right) \supset{ }_{0} Y
$$

there exists a nonzero $B(\mathcal{H})$-bimodule morphism from ${ }_{0} Y$ into $\left(X_{0}\right)^{* * *}$ (e.g., the inclusion mapping). Let $\tau:{ }_{0} Y \rightarrow\left(X_{0}\right)^{* * *}$ be such a morphism, and let $\Delta:\left(X_{0}\right)^{* * *} \rightarrow$ $\left(X_{0}\right)^{*}$ be the projection with the kernel $X_{0}^{\perp}$. Take $T=\Delta \circ \tau:{ }_{0} Y \rightarrow X_{0}^{*}$. From the preceding paragraph, we have that $T=0$. So

$$
\langle x, \tau(y)\rangle=\langle x, T(y)\rangle=0 \quad\left(y \in{ }_{0} Y, x \in X_{0}\right) .
$$

Now let $\Gamma: X_{0} \dot{+}{ }_{0} Y \rightarrow\left(X_{0} \dot{+}{ }_{0} Y\right)^{* * *}$ be the continuous $B(\mathcal{H})$-bimodule morphism defined by

$$
\Gamma((x, y))=(\tau(y), 0) .
$$

Then, for $\left(x_{1}, y_{1}\right),\left(x_{2}, y_{2}\right) \in X_{0} \dot{+}{ }_{0} Y$, and $u \in B(\mathcal{H})^{* *}$, we have

$$
\begin{aligned}
& \left\langle u,\left(x_{1}, y_{1}\right) \cdot \Gamma\left(\left(x_{2}, y_{2}\right)\right)+\Gamma\left(\left(x_{1}, y_{1}\right)\right) \cdot\left(x_{2}, y_{2}\right)\right\rangle \\
& =\left\langle\left(u \cdot x_{1}, 0\right),\left(\tau\left(y_{2}\right), 0\right)\right\rangle+\left\langle\left(0, y_{2} \cdot u\right),\left(\tau\left(y_{1}\right), 0\right)\right\rangle \\
& =\left\langle u \cdot x_{1}, \tau\left(y_{2}\right)\right\rangle=\left\langle u x_{1}, \tau\left(y_{2}\right)\right\rangle=0 .
\end{aligned}
$$


Here we used the fact that $u \cdot x_{1}=u x_{1} \in X_{0}$ (see the paragraph following Lemma (7.3). So

$$
\left(x_{1}, y_{1}\right) \cdot \Gamma\left(\left(x_{2}, y_{2}\right)\right)+\Gamma\left(\left(x_{1}, y_{1}\right)\right) \cdot\left(x_{2}, y_{2}\right)=0
$$

for all $\left(x_{1}, y_{1}\right),\left(x_{2}, y_{2}\right) \in X_{0} \dot{+}{ }_{0} Y$. But $\Gamma \neq 0$; so condition 4 of Theorem 2.1] does not hold for $m=1$ and $X=X_{0} \dot{+}{ }_{0} Y$. As a consequence, $B(\mathcal{H}) \oplus\left(X_{0} \dot{+}{ }_{0} Y\right)$ is not 3 -weakly amenable.

\section{ACKNOWLEDGEMENT}

The author thanks Professor F. Ghahramani, who brought to him the problem of whether weak amenability implies 3-weak amenability and suggested he should look for a counterexample amongst Banach algebras of the form $\mathfrak{A} \oplus X$. He also appreciates valuable discussions about this topic with Professors H. G. Dales, J. Duncan, N. Grønbæk, and G. A. Willis.

\section{REFERENCES}

1. W. G. Bade, P. C. Curtis and H. G. Dales, Amenability and weak amenability for Beurling and Lipschitz algebras, Proc. London Math. Soc. 55 (1987), 359-377. MR 88f:46098

2. W. G. Bade, H. G. Dales and Z. A. Lykova, Algebraic and strong splittings of extensions of Banach algebras, Mem. Amer. Math. Soc. 137, no. 656, 1999. MR 99g:46059

3. F. F. Bonsall and J. Duncan, Complete normed algebras, Springer-Verlag, Berlin, 1973. MR 54:11013

4. A. Brown, P. R. Halmos and C. Pearcy, Commutators of operators on Hilbert space, Canad. J. Math. 17 (1965) 695-708. MR 34:3311

5. A. Brown and C. Pearcy, Commutators in factors of type III, Canad. J. Math. 18 (1966) 1152-1160. MR 34:1864

6. J. B. Conway, A course in functional analysis, Springer-Verlag, New York, $1985 . \quad$ MR 86h:46001

7. P. C. Curtis Jr., Amenability, weak amenability, and the close homomorphism property for commutative Banach algebras, Function spaces, Edwardsville, IL, 1994, 59-69, Lecture Notes in Pure and Appl. Math., 172, Dekker, New York, 1995. MR 96g:46038

8. P. C. Curtis, Jr., and R. J. Loy, The structure of amenable Banach algebras, J. London Math. Soc. 40 (1989), 89-104. MR 90k:46114

9. H. G. Dales, Banach algebras and automatic continuity, Oxford, New York, 2000. MR 2002e: 46001

10. H. G. Dales, F. Ghahramani and N. Grønbæk, Derivations into iterated duals of Banach algebras, Studia Math. 128 (1998), 19-54. MR 99g:46064

11. M. Despic and F. Ghahramani, Weak amenability of group algebras of locally compact groups, Canad. Math. Bull. 37 (1994), 165-167. MR 95c:43003

12. B. E. Forrest and L. W. Marcoux, Weak amenability of triangular Banach algebras, Trans. Amer. Math. Soc. 354 (2002), 1435-1452.

13. F. Ghahramani, R. J. Loy, and G. A. Willis, Amenability and weak amenability of second conjugate Banach algebras, Proc. Amer. Math. Soc. 124 (1996), 1489-1497. MR 96g:46036

14. N. Grønbæk, Weak and cyclic amenability for noncommutative Banach algebras, Proc. Edinburgh Math. Soc. 35 (1992), 315-328. MR 93d:46082

15. N. Grønbæk, Amenability and weak amenability of tensor algebras and algebras of nuclear operators, J. Austral. Math. Soc. Ser. A 51 (1991), 483-488. MR 92f:46062

16. N. Grønbæk, A characterization of weakly amenable Banach algebras, Studia Math. 94 (1989), 149-162. MR 92a:46055

17. U. Haagerup, All nuclear $C^{*}$-algebras are amenable, Invent. Math. 74 (1983), 305-319. MR 85g:46074

18. P. R. Halmos, Commutators of operators, II, Amer. J. Math. 76 (1954), 191-198. MR 15:538d

19. A. Ya. Helemskii, Banach and locally convex algebras, Oxford University Press, Oxford, 1993. MR 94f:46001 
20. E. Hewitt and K. A. Ross, Abstract harmonic analysis II, Springer-Verlag, Berlin, 1970. MR 41:7378

21. B. E. Johnson, Weak amenability of group algebras, Bull. London Math. Soc. 23 (1991), 281-284. MR 92k:43004

22. B. E. Johnson, Derivations from $L^{1}(G)$ into $L^{1}(G)$ and $L^{\infty}(G)$, Harmonic analysis, Luxembourg, 1987, 191-198, Lecture Notes in Math., 1359, Springer, Berlin, 1988. MR 90a:46122

23. B. E. Johnson, Cohomology in Banach algebra, Mem. Amer. Math. Soc. 127, 1972. MR 51:11130

24. A. T. Lau and R. J. Loy, Weak amenability of Banach algebras on locally compact groups, J. Funct. Anal. 145 (1997), 175-204. MR 98h:46068

25. V. Losert, A characterization of SIN-groups, Math. Ann. 273 (1985), 81-88. MR 87j:22011

26. P. Milnes, Uniformity and uniformly continuous functions for locally compact groups, Proc. Amer. Math. Soc. 109 (1990), 567-570. MR 90i:22006

27. T. W. Palmer, Banach algebra and the general theory of *algebras, Vol. I, Cambridge, 1994. MR 95c: 46002

28. T. W. Palmer, Classes of nonabelian, noncompact, locally compact groups, Rocky Mt. J. 8 (1978), 683-741. MR 81j:22003

29. C. Pearcy and D. Topping, On commutators in ideals of compact operators, Michigan Math. J. 18 (1971), 247-252. MR 44:2077

30. Yong Zhang, Nilpotent ideals in a class of Banach algebras, Proc. Amer. Math. Soc., 127 (1999), 3237-3242. MR 2000j:46086

31. Yong Zhang, Weak amenability of a class of Banach algebras, Canad. Math. Bull. 44 (2001), 504-508.

Department of Mathematics, University of Manitoba, Winnipeg, Manitoba, Canada R3T $2 \mathrm{~N} 2$

E-mail address: zhangy@cc.umanitoba.ca 